\title{
High-energy string-brane scattering: leading eikonal and beyond
}

\author{
Giuseppe D’Appollonio, ${ }^{a, b}$ Paolo Di Vecchia, ${ }^{c, d}$ Rodolfo Russo ${ }^{e}$ and \\ Gabriele Veneziano $^{f, g}$ \\ ${ }^{a}$ Dipartimento di Fisica, Università di Cagliari and INFN, \\ Cittadella Universitaria, 09042 Monserrato, Italy \\ ${ }^{b}$ Laboratoire de Physique Théorique de L'Ecole Normale Supérieure, \\ 24 rue Lhomond, 75231 Paris cedex, France \\ ${ }^{c}$ The Niels Bohr Institute, \\ Blegdamsvej 17, DK-2100 Copenhagen, Denmark \\ ${ }^{d}$ Nordita, \\ Roslagstullsbacken 23, SE-10691 Stockholm, Sweden \\ ${ }^{e}$ Queen Mary University of London, \\ Mile End Road, E1 4 NS London, United Kingdom \\ ${ }^{f}$ Collège de France, \\ 11 place M. Berthelot, 75005 Paris, France \\ ${ }^{g}$ Theory Division, CERN, \\ CH-1211 Geneva 23, Switzerland \\ E-mail: giuseppe.dappollonio@ca.infn.it, divecchi@nbi.dk, \\ r.russo@qmul.ac.uk, gabriele.veneziano@cern.ch
}

ABSTRACT: We extend previous techniques for calculations of transplanckian-energy stringstring collisions to the high-energy scattering of massless closed strings from a stack of $N$ Dp-branes in Minkowski spacetime. We show that an effective non-trivial metric emerges from the string scattering amplitudes by comparing them against the semiclassical dynamics of high-energy strings in the extremal p-brane background. By changing the energy, impact parameter and effective open string coupling $\lambda=g N$, we are able to explore various interesting regimes and to reproduce classical expectations, including tidal-force excitations, even beyond the leading-eikonal approximation.

KEYwORDS: Superstrings and Heterotic Strings, D-branes

ARXIV EPRINT: 1008.4773 


\section{Contents}

1 Introduction 1

2 Scattering of a closed string on $N$ Dp-branes 4

$\begin{array}{lll}2.1 & \text { Disk and annulus amplitudes } & 7\end{array}$

2.2 The Field Theory limit at high energies 8

$\begin{array}{ll}2.3 & \text { String corrections to the leading eikonal } \\ \end{array}$

3 Analysis of the amplitude $\quad \mathbf{1 5}$

3.1 Deflection angle up to next-to-leading order 16

$\begin{array}{lll}3.2 & \text { Tidal excitation at leading order } & 17\end{array}$

4 Comparison with curved-spacetime expectations $\quad 19$

4.1 Classical deflection up to next-to-leading order 20

4.2 Tidal excitation of the closed string at leading order 21

5 Conclusions $\quad 24$

$\begin{array}{ll}\text { A The saddle point in the annulus moduli space } & 26\end{array}$

$\begin{array}{ll}\text { B Dilaton scattering in field theory } & 31\end{array}$

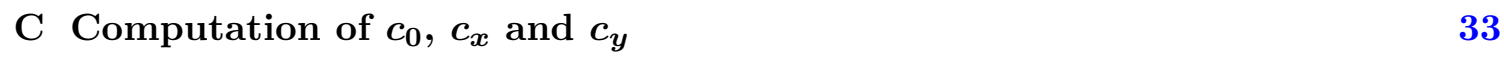

\section{Introduction}

One of the most remarkable properties of string theory is the generic presence, in its massless spectrum, of a spin-2 particle behaving as the graviton of general relativity. Invariance under general coordinate transformations emerges as part of the larger gauge symmetry of string theory and string interactions are automatically compatible with it. This is clearly reflected in the low-energy string effective action, which can be obtained from the scattering amplitudes of the massless excitations and which reduces to an extended - and typically supersymmetric — version of general relativity with additional short-distance corrections. As a result, in string theory spacetime is dynamical, even if the perturbative formulation of the theory requires the choice of a fixed background. Any highly massive or energetic state should then produce physical effects that can be ascribed to an effective spacetime metric.

In a series of papers $[1,3-5]^{1}$ Amati, Ciafaloni and one of us (referred to hereafter as ACV) analysed the way in which classical gravity effects emerge from string computa-

\footnotetext{
${ }^{1}$ See also [6] for a relevant string-loop calculation and $[7,8]$ for other derivations of the leading eikonal in the field theory limit. A different approach [9] is valid at fixed (and even large) angle within a high-energy window. The two methods agree in the intersection of their respective regions of validity.
} 
tions performed in flat Minkowski space and developed methods to derive quantum and string corrections to general relativity. The process considered in ACV is the collision of two strings at trans-planckian energies in a flat Minkowski background. Given the strong gravitational fields generated in the process, one expects that the effective spacetime experienced by the two colliding quanta will be modified in a drastic way, possibly leading to gravitational collapse and black hole formation.

In $[1,2]$ it was shown that an eikonal resummation of the leading high-energy contributions to the S-matrix leads to results that are fully consistent with general relativity expectations whenever effects due to the finite string size $\left(l_{s}=\sqrt{\alpha^{\prime} \hbar}\right)$ can be neglected. In this particular process the effective geometry turns out to be, to leading order, the wellknown Aichelburg-Sexl (AS) shock-wave metric [11] produced by an energetic pointlike massless particle. This simple and intuitive interpretation unfortunately fails beyond the leading-eikonal (or small deflection angle) approximation $[4,5]$. In general the effective geometry produced by the collision is expected to be very complicated and to depend on the system as a whole rather than on each individual colliding string. An all-order approach was proposed in [12] (see also [13]) but it turned out to be too difficult to implement this beyond the leading-eikonal approximation.

Recently there has been some progress [14-18] in trying to compute these "classical corrections" to all orders through a dimensionally-reduced effective action, but the approximations made are not yet fully under control. Nonetheless these approximations seem to lead to physically sensible results, at least in terms of identifying semiquantitatively the critical parameters for gravitational collapse and of comparing them with those based on the formation of a trapped surface [20-23].

Several effects due to a non-vanishing string length $l_{s} \neq 0$ were also computed in $[1$, $3,24]$ : some of them do have a general relativity interpretation (e.g. as the effect of tidal forces exerted by an AS metric on extended objects [25]) while others (like the possible absorption of the elastic channel due to s-channel formation of heavy strings) do not. On the whole, a picture emerges whereby string-size effects prevent gravitational collapse when the Schwarzschild radius of the would-be back hole is smaller than the string length parameter $l_{s}$ while the approach to gravitational collapse is characterized, at the quantum level, by a rapid increase in multiplicity and by the corresponding softening of the final quanta $[14,17,24]$. The transition to the black-hole formation regime, which resembles a phase transition in general relativity, may turn out to be smoother in the quantum case.

In this paper we shall apply the approach developed by ACV to the study of a different process, the scattering of a closed string from a stack of $N$ parallel Dp-branes in Minkowski spacetime. The D-branes are massive solitons for which a microscopic string description is available [28]. This important property makes the string-brane system an ideal framework to understand the way in which string scattering amplitudes evaluated in flat space can provide information about the dynamics in an effective curved spacetime. ${ }^{2}$ Indeed, from

\footnotetext{
${ }^{2}$ There is an analogue of this in quantum field theory: as shown long ago by Duff [26, 27], a class of tree diagrams for the scattering of a test particle from a classical source reproduces the physical effects of the effective Schwarzschild metric generated by the source. The difference is that in string theory we have a microscopic quantum description of the source itself and of its couplings to the test particles.
} 
the point of view of perturbative string theory the presence of a collection of Dp-branes is entirely taken into account by the addition of an open string sector with suitable boundary conditions and does not require any modification of the background. On the other hand, from the point of view of the low-energy effective field theory the Dp-branes are a massive charged state and their presence will necessarily result in a curved spacetime.

The backreaction of the D-brane system on spacetime is expected to be well-described by the extremal p-branes [29], which are BPS solutions of the supergravity equations of motion with a non-trivial metric, dilaton and Ramond-Ramond $(p+1)$-form potential. For $p<7$ and in the string frame the extremal p-brane solution is given by

$$
\begin{aligned}
d s^{2} & =\frac{1}{\sqrt{H(r)}}\left(\eta_{\alpha \beta} d x^{\alpha} d x^{\beta}\right)+\sqrt{H(r)}\left(\delta_{i j} d x^{i} d x^{j}\right), \\
\mathrm{e}^{\phi(x)} & =g[H(r)]^{\frac{3-p}{4}}, \quad \mathcal{C}_{01 \ldots p}(x)=\frac{1}{H(r)}-1,
\end{aligned}
$$

where the indices $\alpha, \beta, \ldots$ run along the Dp-brane world-volume, the indices $i, j, \ldots$ indicate the transverse directions and $r^{2}=\delta_{i j} x^{i} x^{j}$. Finally

$$
H(r)=1+\left(\frac{R_{p}}{r}\right)^{7-p}, \quad R_{p}^{7-p}=\frac{g N\left(2 \pi \sqrt{\alpha^{\prime}}\right)^{7-p}}{(7-p) \Omega_{8-p}}, \quad \Omega_{n}=\frac{2 \pi^{\frac{n+1}{2}}}{\Gamma\left(\frac{n+1}{2}\right)},
$$

where $g$ is the dimensionless string coupling constant, $N$ the number of Dp-branes and $\Omega_{n}$ the volume of the $n$-dimensional unit sphere. This effective description should be reliable as long as the curvature is small in string units. Evidence that $N$ parallel Dp-branes correspond to the curved spacetime given in eqs. (1.1) and (1.2) was provided in [30-32] where it was shown that the large distance behaviour of the classical solutions can be recovered from string-brane scattering amplitudes at tree level.

We shall analyse the system of $N$ parallel Dp-branes using as a probe a high-energy massless closed string. In the high-energy limit the dominant interaction will be gravity and we will not be sensitive to the other background fields. We shall also work in the regime of small string coupling $(g \ll 1)$ and we shall therefore disregard all non-planar diagrams since their contribution is suppressed by powers of $g$. On the other hand, if we keep $\lambda=g N$ fixed (and thus let $N \rightarrow \infty$ ) contributions with many open string loops (boundaries) are not suppressed. This is a welcome feature: indeed, in the regime where the energy $E$ of the closed string is very large the scattering amplitude grows with energy in such a way that partial-wave unitarity breaks down at any finite order. These large contributions to the S-matrix from diagrams with a different number of boundaries are expected to combine together and yield a unitary eikonal form for the resummed amplitude.

As in the case of the string-string scattering, our ultimate aim is to calculate the eikonal phase to all orders in the classical and string corrections. It will turn out that the problem of string scattering from a stack of D-branes is much easier to deal with than the original ACV-type collision problems. Indeed, the former problem is similar to that of scattering in an external field with the closed string acting as a probe and not back-reacting on the geometry. As a result, while the resummation of the closed string loops in the string-string scattering studied in ACV leads to an effective geometry that depends non-trivially upon 
the energies of the two colliding quanta, the metric that emerges from the resummation of open string loops considered in this paper (e.g the length $R_{p}$ defined in (1.3)) depends only on the D-brane system.

Finally, we note that in our approach the existence of a non-trivial background is taken into account by the inclusion of surfaces with boundaries in the perturbative series, rather than by a direct modification of the couplings in the closed string sigma model. Since the string-brane scattering amplitude is evaluated using this microscopic definition of the system, in principle we can also analyse the background generated by $N$ Dp-branes in regions where the effective description (1.1) and (1.2) is not reliable.

The rest of the paper is organised as follows. In section 2 we discuss the high-energy Regge limit of the two-point amplitude of a massless closed string state in the background of $N$ Dp-branes. We analyse in detail the disk and the annulus diagrams both in momentum and in impact-parameter space and then, relying on the results of $[1,2]$, we show that the resummed scattering amplitude has an operator eikonal form. We also compute explicitly the first subleading correction to the eikonal. In section 3 we analyse the S-matrix by a semiclassical saddle point approximation at large impact parameters where one can expect that the description of the string-brane system in terms of a smooth effective background metric should be recovered. In section 4 we show that there is indeed full agreement with the results obtained by a semiclassical analysis of the propagation of a closed string in the extremal p-brane spacetime. In particular, we show that the string computation reproduces the classical deflection angle of a null geodesic to next-to-leading order as well as the inelastic amplitude for the string excitations due to the gravitational tidal forces. In section 5 we discuss, with the help of a phase diagram, the different regimes that have been (or can be) explored in our approach and comment on the relevance of this framework to address some aspects of black hole physics. Finally, in appendix A and B we provide some more details about the string annulus calculation and the corresponding field theory diagrams discussed in section 2. In appendix $\mathrm{C}$ we do the same for the effective action (discussed in section 4) that describes the excitation of a closed string moving in the curved spacetime sourced by the Dp-brane system.

\section{Scattering of a closed string on $N$ Dp-branes}

Closed string two-point amplitudes in the background of $N$ Dp-branes can be evaluated in perturbation theory summing over Riemann surfaces with an arbitrary number of boundaries and handles. In order to isolate the backreaction of the branes on the geometry of the spacetime one can consider the limit in which the open string coupling $\lambda=g N$ is kept fixed while $g \rightarrow 0$ and $N \rightarrow \infty$. The leading contribution then comes from surfaces with an arbitrary number of boundaries and no handles and the resummed amplitude is expected to allow an interpretation in terms of the propagation of a closed string in the curved spacetime of an extremal Dp-brane system, see eq. (1.1).

Our setup is reminiscent of the configuration considered in [33], where a highly energetic massless closed string was used to probe a collection of massive closed strings. The large number of constituents in the target plays in this case the same role as the number of D- 
branes in our case and can be used to suppress the contributions from the closed string loops with respect to the tree diagrams. Of course there are important conceptual and technical differences. For instance, a system of $N$ parallel D-branes preserves 16 supercharges; thus it cannot decay and the various constituents do not interact among themselves. This allows for a precise comparison between the results obtained from the string scattering amplitudes and those derived from the effective p-brane metric.

In this paper we focus on the scattering of a massless closed string belonging to the NS-NS sector. The incoming and the outgoing strings are characterized, respectively, by their momenta $p_{1}$ and $p_{2}$ and their polarization tensors $\epsilon_{1}$ and $\epsilon_{2}$. The relevant vertex operators in the $(-1,-1)$ and $(0,0)$ pictures are

$$
\begin{aligned}
V_{-1}(p, \epsilon) & =\frac{\kappa}{2 \pi} \epsilon_{\mu \nu} e^{-\phi-\bar{\phi}} \psi^{\mu} \bar{\psi}^{\nu} e^{i p X} \\
V_{0}(p, \epsilon) & =-\frac{\kappa}{\pi \alpha^{\prime}} \epsilon_{\mu \nu}\left(i \partial X^{\mu}+\frac{\alpha^{\prime}}{2} p \psi \psi^{\mu}\right)\left(i \bar{\partial} X^{\nu}+\frac{\alpha^{\prime}}{2} p \bar{\psi} \bar{\psi}^{\nu}\right) e^{i p X},
\end{aligned}
$$

where $\kappa^{2}=2^{6} \pi^{7} \alpha^{4} g^{2}$ is the gravitational coupling constant in ten dimensions and we follow the conventions of [34]. Translation invariance along the $p+1$ directions parallel to the brane implies the conservation of energy and momentum along the D-brane world-volume

$$
\left(p_{1}+p_{2}\right)_{\|}=0 .
$$

The scattering amplitude is a function of two invariants of the residual Lorentz group $\mathrm{SO}(1, p) \times \mathrm{SO}(9-p)$. Taking, without loss of generality, $p_{1}$ and $p_{2}$ to have vanishing components parallel to the brane, these can be written as

$$
s=E^{2}=\left|k_{1}\right|^{2}=\left|k_{2}\right|^{2}, \quad t=-\left(k_{1}+k_{2}\right)^{2}=-4 E^{2} \sin ^{2} \frac{\Theta}{2},
$$

where $k=p_{\perp}$ denotes the $(9-p)$-dimensional vector transverse to the world-volume of the Dp-brane and $\Theta$ the angle between $k_{1}$ and $-k_{2}$. The two invariants correspond, respectively, to the energy of the incoming string and to the momentum transferred to the brane. For small deflection angles, $k_{1}$ and $k_{2}$ are nearly (anti)parallel and their difference, $\left(k_{1}-k_{2}\right)$, defines a privileged transverse direction, that of the large external momenta. The impactparameter vector $b$, which lies in the direction of $q \equiv-\left(k_{1}+k_{2}\right)$, is approximately orthogonal to such a direction and, together with it, defines the collision plane. In the following we shall often use bold characters to denote $(8-p)$-dimensional vectors transverse both to the brane worldvolume and to the direction of $\left(k_{1}-k_{2}\right)$.

Besides taking the large- $N$ limit at fixed $g N$, we will also consider this process in the high-energy, small scattering angle limit defined by the conditions

$$
\frac{\alpha^{\prime} s}{\hbar} \gg 1, \quad \Theta \ll 1,
$$

where we have re-introduced momentarily Planck's constant to emphasize that this limit allows for a semiclassical treatment. As already stressed in $[1,2]$, this regime, even if $\Theta$ is kept fixed and therefore $t$ becomes large, is dominated by soft dynamics, i.e. by 
the exchange of many gravitons whose individual transverse momenta are of order (and somewhat smaller than) $R_{p}^{-1} \ll \sqrt{-t}$ and the corresponding relevant impact parameters $b$ are somewhat larger than $R_{p}$. This is the reason why we can justify the use of the Regge limit, order by order in the loop expansion, for the calculation of the scattering amplitude in $b$ space. After resummation at fixed $b$, we can go back to $t$ and verify that we can cover the kinematical region (2.4).

Note that by fixing $g N$ we are also fixing, through eq. (1.3), the ratio $R_{p} / l_{s}$. In the rest of this paper we will consider brane configurations with $R_{p}>l_{s}$, the stringy regime $R_{p}<l_{s}$ will be briefly mentioned only in section 5 . When $R_{p}>l_{s}$ the string-brane interactions are dominated by gravity effects and one expects a more transparent relation with the effective geometry in eqs. (1.1), (1.2). Since the small-angle condition requires $b \gg R_{p}$, we can see that our scattering amplitudes will be evaluated at impact parameters much larger than the string scale, $b \gg l_{s}$. The conditions (2.4) also imply

$$
\frac{R_{p} E}{\hbar} \gg 1, \quad \frac{b E}{\hbar}=\frac{J}{\hbar} \gg 1,
$$

where $J$ is the (conserved) angular momentum.

Finally, we will impose an upper limit on $E$ such that the effects of closed string loops can be neglected. As we shall discuss more quantitatively at the end of this section, such a limit can be arbitrarily large if we go to sufficiently large $N$ (and thus sufficiently small $g$ ). When this condition is fulfilled, the two-point amplitude can be written as follows

$$
A\left(p_{1}, \epsilon_{1} ; p_{2}, \epsilon_{2}\right)=i(2 \pi)^{p+1} \delta^{p+1}\left(p_{1, \|}+p_{2, \|}\right) \sum_{h=1}^{\infty} A_{h}\left(p_{1}, \epsilon_{1} ; p_{2}, \epsilon_{2}\right),
$$

where the label $h$ counts the number of boundaries. Furthermore, in our limit the dependence of the scattering amplitude on the polarization tensors simplifies considerably, the leading term being proportional to $\operatorname{Tr}\left(\epsilon_{1} \epsilon_{2}^{T}\right)$

$$
A_{h}\left(p_{1}, \epsilon_{1} ; p_{2}, \epsilon_{2}\right) \sim \operatorname{Tr}\left(\epsilon_{1} \epsilon_{2}^{\mathrm{T}}\right) \mathcal{A}_{h}(s, t),
$$

where $\mathcal{A}_{h}$ contains only the part of the string amplitude that diverges at high energy. Setting

$$
\mathcal{A}(s, t)=\sum_{h=1}^{\infty} \mathcal{A}_{h}(s, t)
$$

we obtain the following expression for the Regge limit of the T-matrix, related to the S-matrix by $S=1+i T$,

$$
T\left(p_{1}, \epsilon_{1} ; p_{2}, \epsilon_{2}\right) \sim(2 \pi)^{p+1} \delta^{p+1}\left(p_{1, \|}+p_{2, \|}\right) \operatorname{Tr}\left(\epsilon_{1} \epsilon_{2}^{T}\right) \frac{\mathcal{A}(s, t)}{2 E},
$$

where the factor $2 E$ takes into account the correct normalization of the asymptotic states. The first term in the series in eq. (2.6) is the familiar disk amplitude, the second one is the annulus amplitude. We shall first analyse the Regge limit of these two amplitudes and then explain how to obtain the leading behaviour of the full perturbative series (2.6). As in $[1,2]$, the resummed amplitude takes a simple operator eikonal form in impact parameter space. The eikonal operator will allow us to study both the emergence of classical gravity effects from open string loops and the string corrections to the classical dynamics. 


\subsection{Disk and annulus amplitudes}

The amplitude describing the elastic scattering of a massless NS-NS closed string state on the disk is well-known $[30,35,36]^{3}$ and reads

$$
A_{1}\left(p_{1}, \epsilon_{1} ; p_{2}, \epsilon_{2}\right)=-\frac{\pi^{\frac{9-p}{2}} R_{p}^{7-p}}{\Gamma\left(\frac{7-p}{2}\right)} \mathcal{K}\left(p_{1}, \epsilon_{1} ; p_{2}, \epsilon_{2}\right) \frac{\Gamma\left(-\alpha^{\prime} s\right) \Gamma\left(-\frac{\alpha^{\prime}}{4} t\right)}{\Gamma\left(1-\alpha^{\prime} s-\frac{\alpha^{\prime}}{4} t\right)},
$$

where the full kinematical factor $\mathcal{K}\left(p_{1}, \epsilon_{1} ; p_{2}, \epsilon_{2}\right)$ can be found, for instance, in [30]. The amplitude exhibits poles both in the $t$-channel and in the s-channel. The former are due to the exchange of closed strings and appear when the two vertex operators approach each other, the latter are due to the exchange of open strings and appear when the two vertex operators approach the boundary of the disk. In the Regge limit the leading term in the kinematical factor is

$$
\mathcal{K}\left(p_{1}, \epsilon_{1} ; p_{2}, \epsilon_{2}\right) \sim \operatorname{Tr}\left(\epsilon_{1} \epsilon_{2}^{\mathrm{T}}\right)\left(\alpha^{\prime} s\right)^{2} .
$$

Even if in this paper we focus on states belonging to the NS-NS sector, we note that a similar result also holds for the elastic amplitude with two R-R massless fields.

From (2.10) we obtain in the Regge limit

$$
\mathcal{A}_{1}(s, t)=\frac{\pi^{\frac{9-p}{2}} R_{p}^{7-p}}{\Gamma\left(\frac{7-p}{2}\right)} \Gamma\left(-\frac{\alpha^{\prime}}{4} t\right) e^{-i \pi \frac{\alpha^{\prime} t}{4}}\left(\alpha^{\prime} s\right)^{1+\frac{\alpha^{\prime} t}{4}} .
$$

The previous formula shows that the amplitude is dominated by the exchange in the $t$ channel of the Regge trajectory of the graviton. The imaginary part takes into account inelastic processes where the closed string excites open string degrees of freedom attached to the brane worldvolume. As the energy increases the approximation of single-reggeon exchange eventually breaks down and the tree-level amplitude violates unitarity. As it is well-known both in field theory [38, 39] and in string theory $[1,2,8]$, unitarity is recovered by taking into account multi-reggeon exchanges, described in our case by diagrams with a higher number of boundaries.

Let us consider now the amplitude with two boundaries, the second term in the perturbative series in eq. (2.6). In general, perturbative string amplitudes are given in terms of correlation functions of the worldsheet theory integrated over the moduli space of Riemann surfaces with punctures. In the simple case of a surface with two boundaries and two punctures we have, besides the insertion points of the two vertex operators, $z_{1}$ and $z_{2}$, a single purely imaginary modular parameter $\tau_{\mathrm{op}}=i \tau_{2}$. Depending on whether the worldsheet time direction is chosen parallel or orthogonal to the two boundaries, this amplitude can be interpreted either as a one-loop diagram of open strings, the annulus diagram, or as a tree-level diagram of closed strings, the cylinder diagram. The two descriptions are connected by the modular transformation $\tau_{\mathrm{op}} \mapsto \tau_{\mathrm{cl}}=-\frac{1}{\tau_{\mathrm{op}}}$.

As we will show in the following, at large impact parameters the dominant contributions to the scattering amplitude come from the region of large $\lambda=\operatorname{Im}\left(\tau_{\mathrm{cl}}\right)$. For this reason it

\footnotetext{
${ }^{3}$ Disk amplitudes with both open and closed strings were first computed in ref. [37].
} 
is more convenient to represent the amplitude by means of two closed string vertices and two boundary states [40] and to display its explicit form in the closed string channel

$$
A_{2}\left(p_{1}, \epsilon_{1} ; p_{2}, \epsilon_{2}\right)=\mathcal{N} \int d^{2} z_{1} d^{2} z_{2}\left\langle B\left|V_{1}\left(z_{1}, \bar{z}_{1}\right) V_{2}\left(z_{2}, \bar{z}_{2}\right) D\right| B\right\rangle
$$

where $\mathcal{N}$ is a normalization factor and $D$ is the closed string propagator. The final result is (see $[41,42]$ for a derivation of the amplitude and appendix A for our conventions)

$$
\mathcal{A}_{2}(s, t)=\frac{\pi^{3}\left(\alpha^{\prime} s\right)^{2}}{\Gamma^{2}\left(\frac{7-p}{2}\right)} \frac{R_{p}^{14-2 p}}{\left(2 \alpha^{\prime}\right)^{\frac{7-p}{2}}}\left[2 \int_{0}^{\infty} \frac{d \lambda}{\lambda^{\frac{5-p}{2}}} \int_{0}^{\frac{1}{2}} d \rho_{1} \int_{0}^{\frac{1}{2}} d \rho_{2} \int_{0}^{1} d \omega_{1} \int_{0}^{1} d \omega_{2} \mathcal{I}\right]
$$

In the previous formula the dependence on the external momenta is contained in the function

$$
\mathcal{I}=e^{-\alpha^{\prime} s V_{s}-\frac{\alpha^{\prime} t}{4} V_{t}}
$$

which is the correlation function on the annulus of the exponential part of the vertex operators in eq. (2.1). The functions $V_{s}$ and $V_{t}$ can be expressed in terms of the Jacobi theta function $\theta_{1}(z \mid \tau)$ (see eq. (A.1) for the definition) and read

$$
\begin{aligned}
& V_{s}=-2 \pi \lambda \rho^{2}+\log \frac{\theta_{1}(i \lambda(\zeta+\rho) \mid i \lambda) \theta_{1}(i \lambda(\zeta-\rho) \mid i \lambda)}{\theta_{1}(i \lambda \zeta+\omega \mid i \lambda) \theta_{1}(i \lambda \zeta-\omega \mid i \lambda)}, \\
& V_{t}=8 \pi \lambda \rho_{1} \rho_{2}+\log \frac{\theta_{1}(i \lambda \rho+\omega \mid i \lambda) \theta_{1}(i \lambda \rho-\omega \mid i \lambda)}{\theta_{1}(i \lambda \zeta+\omega \mid i \lambda) \theta_{1}(i \lambda \zeta-\omega \mid i \lambda)},
\end{aligned}
$$

where we introduced the variables $\rho=\rho_{1}-\rho_{2}, \zeta=\rho_{1}+\rho_{2}$ and $\omega=\omega_{1}-\omega_{2}$. The amplitude does not depend on $\sigma=\omega_{1}+\omega_{2}$ as a consequence of its invariance under translations in the direction parallel to the boundaries of the cylinder.

\subsection{The Field Theory limit at high energies}

Before presenting the detailed derivation of the high-energy limit of the string amplitudes, which involves several technical points, it is useful to consider the disk and annulus diagrams in the field theory limit. This limit, which corresponds to sending $\alpha^{\prime} \rightarrow 0$ while keeping $R_{p}$ fixed, considerably facilitates the analysis and allows us to illustrate our main results in a simpler setting. The amplitudes discussed below can also be derived by evaluating directly the Feynman diagrams contributing to the scattering process, as described in appendix B.

The field theory limit of the disk amplitude is easily obtained from eq. (2.12) and reads

$$
\mathcal{A}_{1}(s, t) \rightarrow \frac{4 \pi^{\frac{9-p}{2}} R_{p}^{7-p}}{\Gamma\left(\frac{7-p}{2}\right)} \frac{s}{(-t)} .
$$

As expected, at high-energy the amplitude is dominated by the exchange of a single graviton in the $t$-channel.

The field theory limit of the annulus diagram in (2.14) is more subtle since this amplitude is given in terms of an integral over the variables $\lambda, \rho, \zeta$ and $\omega$. It turns out that the variable $T=\alpha^{\prime} \lambda$ plays the role of the Schwinger parameter of the field theory diagrams 
and thus has to be kept fixed in the limit. Since $\alpha^{\prime} \rightarrow 0$, this implies that the relevant region of integration is the region of large values of $\lambda$. We can then rewrite eq. (2.14) in the following way

$$
\mathcal{A}_{2}(s, t) \rightarrow \frac{\pi^{3} s^{2}}{\Gamma^{2}\left(\frac{7-p}{2}\right)} \frac{R_{p}^{14-2 p}}{2^{\frac{7-p}{2}}}\left[\int_{0}^{\infty} \frac{d T}{T^{\frac{5-p}{2}}} \int_{0}^{1} d \zeta \int_{-\zeta}^{\zeta} d \rho \int_{0}^{1} d \omega e^{-\alpha^{\prime} s V_{s}-\frac{\alpha^{\prime} t}{4} V_{t}}\right]
$$

where now $V_{s}$ and $V_{t}$ stand for the asymptotic form at large values of $\lambda$ of the functions in eqs. (2.16) and (2.17), given in eq. (A.2). The first term in $V_{s}$ in (A.2) shows that at high energy the integral over $\rho$ is dominated by the region of integration around $\rho \sim 0$ and therefore we can make the further approximation

$$
\alpha^{\prime} V_{s} \sim-2 \pi T \rho^{2}, \quad \quad \alpha^{\prime} V_{t} \sim-2 \pi T \zeta(1-\zeta)
$$

Substituting in eq. (2.19), we see that the dependance on $\alpha^{\prime}$ disappears from the annulus amplitude. We note that in this limit eq. (2.19) agrees precisely with the field theory integrand for the first Feynman diagram in figure 4 of appendix B, evaluated around the saddle point $y \sim 0$ in the Schwinger parametrization (see eqs. (B.1) and (B.2)).

In order to make the integral over $\rho$ convergent we can continue it analytically to negative values of $E^{2}$ or equivalently we can rotate the integration contour in $\rho$ from the positive real axis to the positive imaginary axis

$$
\int_{-\zeta}^{\zeta} d \rho \mathrm{e}^{2 \pi s T \rho^{2}} \sim \frac{i}{\sqrt{2 T} E}
$$

Evaluating the two remaining integrals over $T$ and $\zeta$ we obtain

$$
\mathcal{A}_{2}^{(3)}(s, t) \rightarrow \frac{\pi^{\frac{9-p}{2}} E^{4}}{\Gamma^{2}\left(\frac{7-p}{2}\right)} \frac{R_{p}^{14-2 p}}{2^{\frac{7-p}{2}}} \frac{i \sqrt{\pi}}{\sqrt{2} E}\left(\frac{2}{|t|}\right)^{\frac{p-4}{2}} \Gamma\left(\frac{p-4}{2}\right) \frac{\Gamma\left(\frac{6-p}{2}\right) \Gamma\left(\frac{6-p}{2}\right)}{\Gamma(6-p)},
$$

where the upper index (3) indicates that this term diverges as $E^{3}$. An alternative expression for the previous amplitude, which will be useful in comparing it with the corresponding full string theory result, is the following

$$
\mathcal{A}_{2}^{(3)}(s, t)=\frac{i}{4 E} \int \frac{d^{8-p} \mathbf{k}}{(2 \pi)^{8-p}}\left(\frac{4 E^{2} R_{p}^{7-p} \pi^{\frac{9-p}{2}}}{\Gamma\left(\frac{7-p}{2}\right)}\right)^{2} \frac{1}{\mathbf{k}^{2}(\mathbf{q}-\mathbf{k})^{2}}, \quad t \equiv-\mathbf{q}^{2}
$$

We see that $\mathcal{A}_{2}^{(3)}$ can be written as the convolution in momentum space of two tree-level amplitudes $\mathcal{A}_{1}$.

The individual perturbative amplitudes, like $\mathcal{A}_{1}$ and $\mathcal{A}_{2}^{(3)}$, are divergent for large values of $E$ and therefore at sufficiently high energy they will violate unitarity. It turns out, however, that the leading contributions in energy coming from the disk, the annulus and the higher loop diagrams, corresponding to surfaces with more than two boundaries, are the terms of an exponential series and combine together to give just a phase in the S-matrix. This is most directly seen if we express the scattering amplitude as a function of the impact 
parameter $b$ instead of $t$. The relation between the S-matrix and the amplitudes computed above is ${ }^{4}$

$$
S=1+i T=1+i \frac{\mathcal{A}}{2 E},
$$

while momentum and impact-parameter space are related by a Fourier transform

$$
T(E, b)=\int \frac{d^{8-p} \mathbf{q}}{(2 \pi)^{8-p}} e^{i \mathbf{b} \cdot \mathbf{q}} T(E, t) .
$$

Using the following equation

$$
\int \frac{d^{D} k}{(2 \pi)^{D}} \mathrm{e}^{i k \cdot b}\left(k^{2}\right)^{\nu}=-\frac{\nu}{\nu+\frac{D}{2}} \frac{2^{2 \nu}}{\pi^{D / 2}} \frac{\Gamma\left(\nu+\frac{D}{2}+1\right)}{\Gamma(1-\nu)} \frac{1}{\left(b^{2}\right)^{\nu+\frac{D}{2}}},
$$

we can transform both eqs. (2.18) and (2.22) to impact-parameter space, getting ${ }^{5}$

$$
i T_{1}(E, b)=i\left(\frac{R_{p}^{7-p} \sqrt{\pi} E}{2 b^{6-p}} \frac{\Gamma\left(\frac{6-p}{2}\right)}{\Gamma\left(\frac{7-p}{2}\right)}\right), \quad i T_{2}(E, b)=-\frac{1}{2}\left(\frac{R_{p}^{7-p} \sqrt{\pi} E}{2 b^{6-p}} \frac{\Gamma\left(\frac{6-p}{2}\right)}{\Gamma\left(\frac{7-p}{2}\right)}\right)^{2} .
$$

In conclusion, we obtain

$$
S(E, b)=1+i T_{1}(E, b)+i T_{2}(E, b)+\cdots=1+i T_{1}(E, b)-\frac{1}{2}\left(T_{1}(E, b)\right)^{2}+\ldots,
$$

which is consistent with the fact that the unitarity violating disk amplitude exponentiates giving just a phase in the S-matrix

$$
S(E, b)=\mathrm{e}^{i T_{1}(E, b)} .
$$

We can now proceed to compute the next-to-leading term in energy in the field theory limit. In this case, as discussed in appendix B, we need to take into account several Feynman diagrams. Their combination reproduces the $\alpha^{\prime} \rightarrow 0$ limit of eq. (A.22) in appendix A

$$
\mathcal{A}_{2}(s, t) \rightarrow \frac{\pi^{3} s}{\Gamma^{2}\left(\frac{7-p}{2}\right)} \frac{R_{p}^{14-2 p}}{2^{\frac{7-p}{2}}} \frac{t}{4} \int_{0}^{\infty} \frac{d T}{T^{\frac{5-p}{2}}} \int_{0}^{1} d \zeta\left[e^{-2 \pi T \zeta(1-\zeta)}\right]^{-\frac{t}{4}} .
$$

This result should be compared with the field theory integrand for diagram (a) in figure 4 of appendix B, where we keep the subleading term in the expansion around the saddle point in the Schwinger parameter $y$. After performing the integrals over $T$ and $\zeta$ in eq. (2.30) we obtain

$$
\mathcal{A}_{2}^{(2)}(s, t)=-\frac{\pi^{\frac{9-p}{2}} E^{2}}{\Gamma^{2}\left(\frac{7-p}{2}\right)} \frac{R_{p}^{14-2 p}}{2^{7-p}|t|^{\frac{p-5}{2}}} \Gamma\left(\frac{p-3}{2}\right) B\left(\frac{5-p}{2}, \frac{5-p}{2}\right) .
$$

\footnotetext{
${ }^{4}$ We omit here for simplicity of notation the terms containing the polarizations of the two external particles and the $\delta$-function required by momentum conservation. The complete expression is given in eq. (2.9).

${ }^{5}$ Here and many times in the following we encounter expressions which exhibit singularities at particular values of $p$. The correct result is obtained by taking the limit from non-integer $p$, as in dimensional regularization, and in throwing away irrelevant infinities. As an example, at $p=6$ eq. (2.27) produces logarithms of $b$ besides an unobservable infinite Coulomb phase.
} 
Using again eq. (2.26), the previous expression becomes in impact parameter space

$$
i T_{2}^{(1)}(E, b) \equiv i \frac{\mathcal{A}_{2}^{(2)}}{2 E}=i \frac{\sqrt{\pi} E R_{p}^{14-2 p}}{b^{13-2 p}} \frac{\Gamma\left(\frac{13-2 p}{2}\right)}{4 \Gamma(6-p)}
$$

The upper indices (2) and (1) denote that $\mathcal{A}_{2}^{(2)}$ and $T_{2}^{(1)}$ diverge respectively as $E^{2}$ and $E$ at high energy. In the next subsection we will generalize these results in order to include the string corrections.

\subsection{String corrections to the leading eikonal}

We now extend the results obtained in the previous subsection in two directions. On the one hand, we evaluate the high-energy limit of the full string amplitude (2.14) in order to derive the string corrections to the leading field theory result; on the other hand, we determine the form of the terms in $\mathcal{A}_{2}$ that scale as $E^{2}$, which are small in comparison to the leading behaviour $E^{3}$, but still yield a divergent contributions to the $T$-matrix (2.9). In the previous section we have already extracted those terms in the field theory limit, but their derivation presented in the appendix includes also some (but possibly not all) string corrections.

The high-energy limit of a string amplitude can be determined by evaluating the asymptotic behaviour of the multidimensional integral over the world-sheet moduli using saddle-point methods [43]. The leading terms are due to critical points in the interior or on the boundary of the integration domain, each critical point corresponding to a specific degeneration limit of a Riemann surface with punctures. We leave the technical steps necessary to evaluate the saddle-points of the annulus with two punctures in eq. (2.14) to the appendix A. Here we just give a sketch of the derivation and summarize the main results.

In the limit (2.4) there are no critical points in the interior of the domain. The dominant contribution comes from the region of small values of $\rho$, since we are taking the high-energy limit for the external states, and from the region of large values of $\lambda$, since we are considering impact parameters larger than the string scale. We shall then expand the integrand for large $\lambda$ and small $\rho$ and perform exactly the integrals over the angular variables $\omega_{1}$ and $\omega_{2}$, which implement the level matching condition. Since we wish to focus on the part of the S-matrix (2.9) that is perturbatively divergent at high energies, we will consider only terms that are at least of order $E^{2}$. It turns out that the expansion of the integrand around $\rho=0$ yields a leading contribution (indicated by $\mathcal{A}_{2}^{(3)}$ ) that scales as $E^{3}$ and a subleading contribution that scales as $E^{2}$ (indicated by $\mathcal{A}_{2}^{(2)}$ ).

The leading term $\mathcal{A}_{2}^{(3)}$ in the annulus amplitude is precisely the one required for the exponentiation of the tree-level amplitude into an operator eikonal form, which generalizes the field theory result given in eq. (2.23) and reconciles the perturbative string expansion with unitarity. More precisely, to all orders in $\alpha^{\prime}$ the leading annulus contribution has the 
following form ${ }^{6}$ (see appendix A for details)

$$
\frac{\mathcal{A}_{2}^{(3)}}{2 E}=\frac{i}{2} \int \frac{d^{8-p} \mathbf{k}}{(2 \pi)^{8-p}} \frac{\mathcal{A}_{1}\left(s, t_{1}\right)}{2 E} \frac{\mathcal{A}_{1}\left(s, t_{2}\right)}{2 E} V_{2}\left(t_{1}, t_{2}, t\right),
$$

where

and

$$
V_{2}\left(t_{1}, t_{2}, t\right)=\frac{\Gamma\left[1+\frac{\alpha^{\prime}}{2}\left(t_{1}+t_{2}-t\right)\right]}{\Gamma^{2}\left[1+\frac{\alpha^{\prime}}{4}\left(t_{1}+t_{2}-t\right)\right]},
$$

$$
\mathbf{q}^{2}=-t, \quad t_{1}=-\mathbf{k}^{2}, \quad t_{2}=-(\mathbf{q}-\mathbf{k})^{2} .
$$

The function $V_{2}\left(t_{1}, t_{2}, t\right)$ is the vertex for the emission of two reggeized gravitons derived in $[1,2]$ in the context of high-energy string-string collisions. The fact that precisely the same vertex appears in the string-brane scattering process studied in this paper is not surprising. As in the field theory description of the deflection of a particle by an external potential, one expects that the string will interact with the brane through multiple exchanges of states lying on the Regge trajectory of the graviton. The leading term of the annulus amplitude confirms this expectation showing that the amplitude factorizes into the product of the vertex for the emission of two reggeized gravitons and two disk amplitudes which encode the boundary conditions pertaining to the brane source.

In the $\alpha^{\prime} t \rightarrow 0$ limit the string amplitude (2.33) reduces to the leading field theory result (2.23) of section 2.2 and a comparison between the two expressions helps to clarify the effect of the string corrections. We see that the graviton pole is replaced by the complete Regge trajectory of the graviton and that the convolution of the tree-level amplitudes now involves a non-trivial kernel $V_{2}\left(t_{1}, t_{2}, t\right)$. As it stands, it is not evident that (2.33) is the second term in the expansion of a simple eikonal form. In order to better understand the structure of the series let us consider the leading terms provided by the higher-order amplitudes.

For a generic amplitude $\mathcal{A}_{h}$ with $h$ boundaries, the term with the highest power of $E$ should correspond to the exchange of $h$ reggeons and scale as $E^{h+1}$. We can then write

$$
\frac{\mathcal{A}_{h}^{(h+1)}(s, t)}{2 E} \sim \frac{1}{h !} \frac{i^{h-1}}{(2 E)^{h}} \prod_{i=1}^{h-1} \int \frac{d^{8-p} \mathbf{k}_{i}}{(2 \pi)^{8-p}} \mathcal{A}_{1}\left(s, t_{1}\right) \ldots \mathcal{A}_{1}\left(s, t_{h}\right) V_{h}\left(\mathbf{k}_{1}, \mathbf{k}_{2}, \ldots, \mathbf{k}_{h}\right),
$$

where $t_{i} \equiv-\mathbf{k}_{i}^{2}$ for $i=1 \ldots h$ and $\sum_{i=1}^{h} \mathbf{k}_{\mathbf{i}}=\mathbf{q}$ generalize the conventions in (2.35), while the vertex for the emission of $h$ reggeized gravitons $V_{h}$ [1,2] generalizes the $h=2$ expression in (2.34). To resum all the leading contributions in eq. (2.36), it is important to realise that the vertices $V_{h}$ have a simple representation in terms of vacuum expectation values of string vertex operators $[1,2]$

$$
V_{h}\left(\mathbf{k}_{1}, \ldots, \mathbf{k}_{h}\right)=\left\langle 0\left|\prod_{i=1}^{h} \int_{0}^{2 \pi} \frac{d \sigma_{i}}{2 \pi}: e^{i \mathbf{k}_{i} \hat{X}\left(\sigma_{i}\right)}:\right| 0\right\rangle,
$$

\footnotetext{
${ }^{6}$ Strictly speaking (2.33) is not well defined because there are non-physical divergences, due to the poles of the gamma function in $V_{2}$, whenever $\mathbf{k}$ is such that $\alpha^{\prime}\left(t_{1}+t_{2}-t\right)=-2,-4, \ldots$ These divergences, however, arise from a region where the integrand is suppressed by factors of $E$. As shown in the appendix A, the full amplitude is free of unphysical poles.
} 
where the string fields $\hat{X}(\sigma)$ are defined in (A.17). By using this operator form for the vertex $V_{h}$, we can rewrite the integrand in (2.36) as a convolution in momentum space and then diagonalize the full string expression going to impact parameter space

$$
\begin{aligned}
i \frac{\mathcal{A}_{h}^{(h+1)}(s, \mathbf{b})}{2 E} & =\int \frac{d^{8-p} \mathbf{q}}{(2 \pi)^{8-p}} e^{i \mathbf{b q}} i \frac{\mathcal{A}_{h}^{(h+1)}(s, t)}{2 E} \\
& =\frac{i^{h}}{h !}\left\langle 0\left|\prod_{i=1}^{h} \int_{0}^{2 \pi} \frac{d \sigma_{i}}{2 \pi} \int \frac{d^{8-p} \mathbf{k}_{i}}{(2 \pi)^{8-p}} \frac{\mathcal{A}_{1}\left(s,-\mathbf{k}_{i}^{2}\right)}{2 E}: \mathrm{e}^{i \mathbf{k}_{i}\left(\mathbf{b}+\hat{X}\left(\sigma_{i}\right)\right)}:\right| 0\right\rangle .
\end{aligned}
$$

Summing the contribution of all the $h$-reggeon diagrams we finally obtain the leading highenergy behaviour of the series in eq. (2.8)

$$
\sum_{h=1}^{\infty} \frac{\mathcal{A}_{h}^{(h+1)}(s, \mathbf{b})}{2 E} \sim\left\langle 0\left|\frac{1}{i}\left[e^{2 i \hat{\delta}(s, b)}-1\right]\right| 0\right\rangle,
$$

where

$$
2 \hat{\delta}(s, b)=\int_{0}^{2 \pi} \frac{d \sigma}{2 \pi} \int \frac{d^{8-p} \mathbf{k}}{(2 \pi)^{8-p}} \frac{\mathcal{A}_{1}\left(s,-\mathbf{k}^{2}\right)}{2 E}: e^{i \mathbf{k}(\mathbf{b}+\hat{\mathbf{X}}(\sigma))}:=\int_{0}^{2 \pi} \frac{d \sigma}{2 \pi} \frac{\mathcal{A}_{1}(s, \mathbf{b}+\hat{\mathbf{X}}(\sigma)):}{2 E} .
$$

As anticipated, the eikonal form of the amplitude is the one expected in a high-energy process dominated by the exchange of reggeized gravitons between the string and the branes. The string eikonal differs from the field theory eikonal in that it involves the full string amplitude (2.12) and the impact parameter is shifted by the string position operator. It is this simple shift that takes into account effects due to the finite string size which can become relevant already at large impact parameters, as we shall explain in the next section.

We now turn to the subleading term $\mathcal{A}_{2}^{(2)}$ in the annulus amplitude (2.14). This subleading contribution has the same energy dependence as the tree-level amplitude (2.12) and its natural interpretation is that it induces a renormalization of the tree-level result

$$
\mathcal{A}_{1}(s, t) \mapsto \mathcal{A}^{(2)}(s, t) \equiv \mathcal{A}_{1}(s, t)+\mathcal{A}_{2}^{(2)}(s, t)+\ldots
$$

where the index (2) denotes that all the terms in the previous series diverge as $E^{2}$ at high energy. The correction to the Regge pole term remains non-trivial also in the field theory limit and it is crucial to reproduce the precise form of the Dp-brane solution in eq. (1.1). For instance, in section 4 we will compute the deflection angle of a null geodesic in the Dp-brane space-time and compare it with the string result in the limit of large impact parameters $b$. In this example one can see explicitly that the one-loop shift $\mathcal{A}_{2}^{(2)}$ yields a non-vanishing contribution to the deflection angle which accounts for the classical sub-leading corrections in $R_{p} / b$.

The sub-leading contributions are computed in appendix A. In particular in eq. (A.23) we give the complete expression for the term which is dominant in the $\alpha^{\prime} t \rightarrow 0$ limit. It is this term that, when multiplied with the prefactor in eq. (2.14), reduces in the field theory 
limit to

$$
\mathcal{A}_{2}^{(2)}(s, t) \rightarrow-s \frac{\pi^{\frac{9-p}{2}}}{2^{7-p}} \frac{B\left(\frac{5-p}{2}, \frac{5-p}{2}\right) \Gamma\left(\frac{p-3}{2}\right)}{\Gamma^{2}\left(\frac{7-p}{2}\right)} \frac{R^{14-2 p}}{(-t)^{\frac{5-p}{2}}},
$$

the expression already given in eq. (2.31).

Let us briefly comment on the subleading terms in the higher-order (many boundary) amplitudes. Besides the leading terms $\mathcal{A}_{h}^{(h+1)}$ which give the eikonal operator in eq. (2.39), string diagrams with $h$ boundaries should also contain contributions of type $\mathcal{A}_{h}^{(2)}$, that is with the same energy dependence as the tree-level amplitude (2.12). These terms are similar to the term $\mathcal{A}_{2}^{(2)}$ just discussed and should provide additional renormalizations of the Regge pole term to be included in (2.41). Clearly there should also be several other terms scaling with a power of the energy intermediate between $\mathcal{A}_{h}^{(h+1)}$ and $\mathcal{A}_{h}^{(2)}$. All these terms should combine to define a high-energy S-matrix which generalizes the leading eikonal operator (2.39), including both subleading classical corrections in powers of $R_{p} / b$ and string corrections in powers of $\alpha^{\prime} / b^{2}$. Exponentiation of the former is necessary in order to reproduce the classical curved spacetime expectations discussed in section 4 . In order to determine the form of the latter one should perform a detailed analysis of the higher-order amplitudes which is beyond the scope of this investigation. Nonetheless, some indications about these string corrections to higher-order terms in the $R_{p} / b$ expansion should be provided by generalizing our study of string propagation in the D-brane metric (1.1) presented in section 4 .

To conclude this section let us go back to the conditions under which we can neglect higher-genus topologies, i.e. closed string loops generating extra handles. Since closed strings live in the bulk any extra closed-string loop should involve the ten-dimensional Newton constant $G_{10} \sim \kappa_{10}^{2} \sim g^{2}\left(\alpha^{\prime}\right)^{4}$ with no associated power of $N$. This is the reason we have already advocated to argue that, at sufficiently large $N$, closed-string loops can be neglected. In order to make the argument more quantitative let us notice that, in analogy with the ACV case, each extra handle can contribute to the eikonal phase at most one extra power of the asymptotic-energy variable, which is $E$ in the present case. Hence, by dimensional arguments, each extra handle will be associated with a factor $\frac{G_{10} E}{b^{7}}$. In general, from a surface with $n$ boundaries and $m$ handles we expect a contribution to the eikonal phase of the form

$$
\delta(n, m) \sim \frac{E b}{\hbar}\left(\frac{R_{p}}{b}\right)^{n(7-p)}\left(\frac{G_{10} E}{b^{7}}\right)^{m} \sim \frac{E b}{\hbar}\left(\frac{E}{M_{s}}\right)^{m}(g N)^{n} g^{2 m}\left(\frac{l_{s}}{b}\right)^{(7-p) n+7 m} .
$$

The first correction to the disc amplitude (2.27) will thus be

$$
\delta(1,1) \sim \frac{E b}{\hbar}\left(\frac{R_{p}}{b}\right)^{7-p}\left(\frac{G_{10} E}{b^{7}}\right) \sim \frac{E b}{\hbar} \frac{E}{M_{s}} g^{3} N\left(\frac{l_{s}}{b}\right)^{14-p} .
$$

On the basis of (2.44) we see that the contribution of one extra handle is negligible if

$$
\frac{E}{M_{s}} \ll N f\left(\frac{R_{p}}{l_{s}}, \frac{R_{p}}{b}\right),
$$


where $f$ is a simple function of its arguments. This condition is obviously satisfied at fixed $R_{p} / l_{s}$ (i.e. fixed $g N$ ) and fixed $R_{p} / b$ (i.e. fixed $\Theta$ ) if $N$ is taken to be sufficiently large. It is easy to see that, under this condition, we also have $\delta(n, m) \ll 1$. Note finally that, given the above-mentioned relation between $G_{10}$ and $\alpha^{\prime}$, the condition (2.45) allows for $E$ to be arbitrarily large even with respect to the 10-dimensional Planck mass.

\section{Analysis of the amplitude}

The eikonal operator derived in the previous section

$$
S(s, \mathbf{b})=e^{2 i \hat{\delta}(s, \mathbf{b})}, \quad 2 \hat{\delta}(s, \mathbf{b})=\frac{1}{2 E} \int \frac{d \sigma}{2 \pi}: \mathcal{A}_{1}(s, \mathbf{b}+\hat{\mathbf{X}}(\sigma)):,
$$

gives the leading behaviour, at high energy and in a series expansion in powers of $R_{p} / b$, of the scattering amplitude of a massless closed string on a stack of $N$ Dp-branes. This amplitude, which resums the dominant contributions of an infinite number of open string loops in Minkowski space, should be interpreted as describing the semiclassical propagation of a closed string in a curved spacetime, the background generated by the D-branes.

In this section we shall use eq. (3.1) to analyse the way in which this curved background influences the motion of our probe string and to obtain some information on the background itself. In our approach we do not assume any knowledge of the effective metric generated by the D-branes, rather we see it emerging dynamically from the resummation of the open string loops. The fact that all-order perturbative string computations in flat space give rise to effects which can be interpreted as due to the motion of the string in an effective curved background was first discussed in the context of string-string collisions at transplanckian energies $[1,2,44,45]$. As explained in the introduction, our results provide a simpler instance of the same phenomenon.

Since the process we are considering is the scattering of an extended object in an asymptotically flat spacetime, there are two main effects we can study: the deflection of the trajectory of the projectile and the excitation of its internal degrees of freedom. As we will show, these two effects are neatly taken into account by the eikonal operator (3.1).

In the next section we will provide a direct derivation of the deflection angle and the excitation spectrum of a string in the extremal p-brane background of eqs. (1.1), (1.2), finding precise agreement with the results of this section. The agreement between the resummation of open string loops in Minkowski space and the quantization of the string in an external metric confirms that, for large $R_{p}$, a collection of $N$ coincident Dp-branes is well approximated by the extremal p-brane solution of the supergravity equations of motion. It also shows that, at least in certain limits, our approach provides a quantitative tool to study the classical dynamics of a string in a curved spacetime. The advantage of the present approach, based on the microscopic and manifestly unitary D-brane description, is that it does not rely on the existence of an effective external metric and can be used to analyse other interesting dynamical regimes, as discussed in section 5 . 
In order to derive from (3.1) both the deflection angle and the excitation spectrum, we expand the eikonal phase in a power series in the string position operators $\hat{X}^{i}$

$$
2 \hat{\delta}(s, \mathbf{b}+\hat{\mathbf{X}}) \sim \frac{1}{2 E}\left[\mathcal{A}_{1}(s, b)+\frac{1}{2} \frac{\partial^{2} \mathcal{A}_{1}(s, b)}{\partial b^{i} \partial b^{j}} \overline{\hat{X}^{i} \hat{X}^{j}}+\ldots\right],
$$

assuming $b \gg R_{p} \gg l_{s} \sqrt{\ln \left(\alpha^{\prime} s\right)}$ and keeping only the first two non-trivial terms. The indices $i, j$ label the $8-p$ directions of the impact parameter space transverse to the brane and to the collision axis and the symbol $\bar{A}$ denotes the average of a local operator $A(\sigma, \tau)$ on the worldsheet

$$
\bar{A} \equiv \frac{1}{2 \pi} \int_{0}^{2 \pi} d \sigma: A(\sigma, \tau=0): .
$$

The two terms in (3.2) will give rise respectively to the leading contribution to the deflection angle and to the leading contribution to the tidal excitation of the string modes. Let us analyse these two effects in turn.

\subsection{Deflection angle up to next-to-leading order}

The first term in (3.2) is simply the Fourier transform of the disk amplitude (2.12)

$$
\mathcal{A}_{1}(s, b) \sim s \sqrt{\pi} \frac{\Gamma\left(\frac{6-p}{2}\right)}{\Gamma\left(\frac{7-p}{2}\right)} \frac{R_{p}^{7-p}}{b^{6-p}}+\frac{i \pi \sqrt{s}}{\Gamma\left(\frac{7-p}{2}\right)} \sqrt{\frac{\pi \alpha^{\prime} s}{\ln \alpha^{\prime} s}}\left(\frac{R_{p}}{l_{s}(s)}\right)^{7-p} e^{-\frac{b^{2}}{l_{s}^{2}(s)}}
$$

where $l_{s}(s)$ is the effective string length, the size of a string of energy $E=\sqrt{s}$

$$
l_{s}(s)=l_{s} \sqrt{\ln \alpha^{\prime} s} .
$$

The previous formula shows that when $b \gg R_{p}$ and $R_{p} \gg l_{s}(s)$ the eikonal phase is predominantly real, since the absorption effects due to the imaginary part in (3.4) becomes relevant only for $b \leq l_{s}(s)$. In the computation of the deflection angle $\Theta_{p}$ we can then approximate $\mathcal{A}_{1}$ with its real part.

The functional relation between the deflection angle and the impact parameter can be derived evaluating the Fourier transform of eq. (3.1) back to momentum space

$$
S(s, t) \sim \int d^{8-p} \mathbf{b} e^{-i \mathbf{b q}} e^{\frac{i}{2 E} \mathcal{A}_{1}(s, b)} .
$$

The integral is dominated by the saddle point

$$
\mathbf{q}=\frac{1}{2 E} \frac{1}{b} \frac{\partial \mathcal{A}_{1}}{\partial b} \mathbf{b}
$$

and, using the small-angle relation $\Theta_{p} \sim-\frac{\mathbf{q} \cdot \widehat{\mathbf{b}}}{E}$, we obtain

$$
\Theta_{p}=-\frac{1}{2 s} \frac{\partial \mathcal{A}_{1}}{\partial b}(s, b) .
$$

In our analysis in section 2 , we showed that the annulus amplitude contains a term, $\mathcal{A}_{2}^{(2)}$, which has the same energy dependence as $\mathcal{A}_{1}$ but is of higher order in $R_{p} / b$. To determine 
how these additional term enters in the eikonal operator in eq. (3.1) would require a more detailed study of the subleading contributions coming from surfaces with more than two boundaries. It is however plausible, at least if one neglects the effect of string corrections, that also this term will exponentiate and contribute to the series expansion of the eikonal phase in powers of $R_{p} / b$. If we make this assumption, we can include in the real part of the eikonal phase in (3.8) the one-loop renormalization of the Regge pole obtaining

$$
\operatorname{Re}\left[\mathcal{A}_{1}+\mathcal{A}_{2}^{(2)}\right] \sim s \sqrt{\pi} \frac{\Gamma\left(\frac{6-p}{2}\right)}{\Gamma\left(\frac{7-p}{2}\right)} \frac{R_{p}^{7-p}}{b^{6-p}}+s \frac{\sqrt{\pi}}{4 \Gamma\left(\frac{7-p}{2}\right)} \frac{\Gamma\left(\frac{13-2 p}{2}\right) \Gamma\left(\frac{5-p}{2}\right)}{\Gamma(5-p)} \frac{R_{p}^{14-2 p}}{b^{13-2 p}} .
$$

Our string computation then leads to the following approximation for the deflection angle of a null geodesic in the background of $N$ Dp-branes

$$
\Theta_{p}=\sqrt{\pi}\left[\frac{\Gamma\left(\frac{8-p}{2}\right)}{\Gamma\left(\frac{7-p}{2}\right)}\left(\frac{R_{p}}{b}\right)^{7-p}+\frac{1}{2} \frac{\Gamma\left(\frac{15-2 p}{2}\right)}{\Gamma(6-p)}\left(\frac{R_{p}}{b}\right)^{2(7-p)}+O\left(\left(\frac{R_{p}}{b}\right)^{3(7-p)}\right)\right] .
$$

In the next section we will show that this result is in perfect agreement with the deflection angle predicted by classical gravity in the extremal p-brane background, giving direct evidence in favour of our assumption that also the subleading term exponentiates.

\subsection{Tidal excitation at leading order}

We now turn to the excitations of the internal degrees of freedom of the string. They are taken into account by the second term in eq. (3.2), which is of leading order both in $R_{p} / b$ and in $\alpha^{\prime} / b^{2}$. The higher-derivative terms in the Taylor expansion of the eikonal phase in eq. (3.1) lead to higher string corrections weighted by the same leading power of $R_{p} / b$ but of higher order in $\alpha^{\prime} / b^{2}$ and are therefore suppressed when $b \gg l_{s}$. It is the term with two derivatives that provides the leading contribution to the imaginary part of the eikonal phase, although it gives a negligible correction to its real part and therefore to the deflection angle. As we shall show it gives rise to absorption effects which, unlike those due to the imaginary part of the tree-level amplitude in eq. (3.4), become important already at large distances $b \gg l_{s}(s)[1,2]$. This imaginary part of the eikonal phase accounts for the excitation of the string under the influence of the long-range gravitational field of the brane [25].

In order to study this effect we derive the explicit form of the eikonal operator (3.1) in terms of the standard operators $\alpha_{n}^{i}, \bar{\alpha}_{n}^{i}, n \in \mathbb{Z}$, corresponding to the left-moving and rightmoving modes in the expansion (A.17) of the fields $\hat{X}^{i}$. Since the eikonal phase depends only on the modulus of the impact parameter, the matrix of its second derivatives has the following simple structure

$$
\frac{1}{4 \sqrt{s}} \frac{\partial^{2} \mathcal{A}_{1}(s, b)}{\partial b_{i} \partial b_{j}}=Q_{\perp}(s, b)\left[\delta_{i j}-\frac{b_{i} b_{j}}{b^{2}}\right]+Q_{\|}(s, b) \frac{b_{i} b_{j}}{b^{2}}
$$

with $7-p$ coincident eigenvalues associated with the components of the string operator orthogonal to the impact parameter

$$
Q_{\perp}(s, b)=\frac{1}{4 \sqrt{s}} \frac{1}{b} \frac{d \mathcal{A}_{1}(s, b)}{d b},
$$


and one eigenvalue associated with the component parallel to it

$$
Q_{\|}(s, b)=\frac{1}{4 \sqrt{s}} \frac{d^{2} \mathcal{A}_{1}(s, b)}{d b^{2}} .
$$

The average of the square of the string coordinates can be expressed in the following convenient form $[1,2]$

$$
\overline{\left(\hat{X}^{i}\right)^{2}}=\alpha^{\prime} \sum_{n=1}^{\infty} \frac{1}{n}\left[2 T_{0, n}^{i}-1-T_{+, n}^{i}-T_{-, n}^{i}\right], \quad i=p+1, \ldots, 8,
$$

where the operators $T_{\alpha, n}^{i}$ are

$$
T_{ \pm, n}^{i}=\frac{1}{n} \alpha_{\mp n}^{i} \bar{\alpha}_{\mp n}^{i}, \quad 2 T_{0, n}^{i}=1+\frac{1}{n}\left(\alpha_{-n}^{i} \alpha_{n}^{i}+\bar{\alpha}_{-n}^{i} \bar{\alpha}_{n}^{i}\right)
$$

and satisfy the following commutation relations

$$
\left[T_{-, n}^{i}, T_{+, m}^{j}\right]=2 T_{0, n}^{i} \delta_{i j} \delta_{n m}, \quad\left[T_{0, n}^{i}, T_{ \pm, m}^{j}\right]= \pm T_{ \pm, n}^{i} \delta_{i j} \delta_{n m} .
$$

The previous relations imply that the operators $T_{\alpha, n}^{i}$ form a $S U(1,1)$ algebra whenever they carry the same $n$ and $i$ labels, otherwise they commute. To quadratic order in the string oscillators the eikonal operator reads

$$
e^{2 i \hat{\delta}(s, \mathbf{b})} \sim e^{\frac{i}{2 \sqrt{s}} \mathcal{A}_{1}(s, b)} \prod_{n=1}^{\infty} \prod_{k=p+1}^{8} e^{i \frac{\alpha^{\prime}}{n} Q_{k}\left(2 T_{0, n}^{k}-1-T_{+, n}^{k}-T_{-, n}^{k}\right)},
$$

where $Q_{k}$ stands for $Q_{\perp}$ or $Q_{\|}$according to whether the direction $k$ is perpendicular or parallel to the impact parameter. Using the identity

$$
e^{x\left(2 T_{0}-T_{+}-T_{-}\right)}=e^{-\frac{x}{1-x} T_{+}} e^{-2 \ln (1-x) T_{0}} e^{-\frac{x}{1-x} T_{-}},
$$

we can rewrite the eikonal operator in normal ordered form

$$
\begin{aligned}
e^{2 i \hat{\delta}(s, \mathbf{b}) \sim} & e^{\frac{i}{2 \sqrt{s}} \mathcal{A}_{1}(s, b)} \prod_{n=1}^{\infty} \prod_{k=p+1}^{8} \frac{e^{-\frac{i \alpha^{\prime} Q_{k}}{n}}}{1-\frac{i \alpha^{\prime} Q_{k}}{n}} \\
& \prod_{n=1}^{\infty} \prod_{k=p+1}^{8} e^{-\frac{i \alpha^{\prime} Q_{k}}{n-i \alpha^{\prime} Q_{k}} T_{+, n}^{k}} e^{-\ln \left(1-\frac{i \alpha^{\prime} Q_{k}}{n}\right)\left(2 T_{0, n}^{k}-1\right)} e^{-\frac{i \alpha^{\prime} Q_{k}}{n-i \alpha^{\prime} Q_{k}} T_{-, n}^{k}} .
\end{aligned}
$$

It is now immediate to evaluate the elastic scattering amplitude, which is given by the vacuum expectation value of the eikonal operator

$$
\left\langle 0\left|e^{2 i \hat{\delta}(s, \mathbf{b})}\right| 0\right\rangle \sim e^{\frac{i}{2 \sqrt{s}} \mathcal{A}_{1}(s, b)} e^{-i \alpha^{\prime} \gamma\left((7-p) Q_{\perp}+Q_{\|}\right)} \Gamma\left(1-i \alpha^{\prime} Q_{\|}\right) \Gamma^{7-p}\left(1-i \alpha^{\prime} Q_{\perp}\right),
$$

where we used the infinite-product representation of the gamma function and $\gamma$ is the EulerMascheroni constant. From the previous formula one can see that the string corrections to the real part of the eikonal phase are negligible at large impact parameters. Their most 
important effect in this limit is to induce a non-vanishing imaginary part which, after using the identity $\Gamma(1+i x) \Gamma(1-i x)=\frac{\pi x}{\sinh \pi x}$, can be written as follows

$$
\left|\left\langle 0\left|e^{2 i \hat{\delta}(s, \mathbf{b})}\right| 0\right\rangle\right| \sim e^{-\frac{1}{2 \sqrt{s}} \operatorname{Im} \mathcal{A}_{1}(s, b)}\left[\frac{\pi \alpha^{\prime} Q_{\perp}}{\sinh \pi \alpha^{\prime} Q_{\perp}}\right]^{\frac{7-p}{2}}\left[\frac{\pi \alpha^{\prime} Q_{\|}}{\sinh \pi \alpha^{\prime} Q_{\|}}\right]^{\frac{1}{2}} .
$$

At high energy the previous formula becomes

$$
\begin{aligned}
\left|\left\langle 0\left|e^{2 i \hat{\delta}(s, \mathbf{b})}\right| 0\right\rangle\right| \sim & e^{-\frac{1}{2 \sqrt{s}} \operatorname{Im} \mathcal{A}_{1}(s, b)}\left(2 \pi \alpha^{\prime}\right)^{\frac{8-p}{2}}\left|Q_{\perp}(s, b)\right|^{\frac{7-p}{2}}\left|Q_{\|}(s, b)\right|^{\frac{1}{2}} \\
& e^{-\frac{\pi}{2} \alpha^{\prime}\left[(7-p)\left|Q_{\perp}(s, b)\right|+\left|Q_{\|}(s, b)\right|\right]} .
\end{aligned}
$$

Using the explicit form of $\mathcal{A}_{1}(s, b)$ we obtain

$$
Q_{\perp}(s, b) \equiv Q_{1}(s, b)=-\frac{\sqrt{\pi}}{2} \sqrt{s} \frac{\Gamma\left(\frac{8-p}{2}\right)}{\Gamma\left(\frac{7-p}{2}\right)} \frac{R^{7-p}}{b^{8-p}}, \quad Q_{\|}(s, b)=-(7-p) Q_{1}(s, b) .
$$

The expression for the elastic amplitude simplifies

$$
\begin{aligned}
\left\langle 0\left|e^{2 i \hat{\delta}(s, \mathbf{b})}\right| 0\right\rangle & \sim e^{\frac{i}{2 \sqrt{s}} \mathcal{A}_{1}(s, b)} \Gamma\left(1+i \alpha^{\prime}(7-p) Q_{1}\right) \Gamma^{7-p}\left(1-i \alpha^{\prime} Q_{1}\right) \\
\left|\left\langle 0\left|e^{2 i \hat{\delta}(s, \mathbf{b})}\right| 0\right\rangle\right| & \sim e^{-\frac{1}{2 \sqrt{s}} \operatorname{Im} \mathcal{A}_{1}(s, b)}\left(2 \pi \alpha^{\prime}\left|Q_{1}(s, b)\right|\right)^{\frac{8-p}{2}} \sqrt{7-p} e^{-\pi \alpha^{\prime}(7-p)\left|Q_{1}(s, b)\right|},
\end{aligned}
$$

and we can see that the absorption of the elastic channel due to string excitations becomes non negligible for $b \leq b_{D}$ where

$$
b_{D}^{8-p}=\frac{\pi}{2} \alpha^{\prime} \sqrt{\pi s}(7-p) \frac{\Gamma\left(\frac{8-p}{2}\right)}{\Gamma\left(\frac{7-p}{2}\right)} R_{p}^{7-p} .
$$

At large distances this effect is more important than the inelastic absorption given by (3.4) and becomes relevant already at impact parameters large compared with both the string scale and the curvature of the brane background. In the next section we will show that the tidal excitations of the string given by (3.25) agree with the results of a semiclassical computation in the Dp-brane spacetime.

\section{Comparison with curved-spacetime expectations}

In this section we will compare the results we have obtained from string computations in Minkowski spacetime to what we would expect from the propagation of point or string-like objects in the non-trivial background (1.1), (1.2) generated by the D-branes. Note that, in the high-energy limit, our probes are only sensitive to the metric part of the background (since gravitational couplings are proportional to the energy), while the dilaton and the RR-form backgrounds would provide subleading corrections not considered in this paper.

We will focus our attention on the comparison of two effects:

- Deflection angles up to next-to-leading order in the point-particle limit.

- Tidal excitation of stringy probes at leading order.

As we shall see, there is full agreement on both effects between the string-loop calculations and curved-spacetime expectations. 


\subsection{Classical deflection up to next-to-leading order}

Let us start by a (quite standard) computation of the deflection suffered by a massless point-like probe in the metric (1.1) produced by our stack of Dp-branes. We shall use a slightly more general form of the metric allowing one to change conformal frame (e.g. from the string frame to the Einstein frame). Since the coordinates involved in the geodesics are only the time $t$ and the spatial coordinates $r$ and $\theta$ of the plane in which the motion takes place, we can limit ourselves to the following part of the metric

$$
d s^{2}=-\alpha(r) d t^{2}+\beta(r)\left(d r^{2}+r^{2} d \theta^{2}\right) .
$$

Since the metric does not depend on the time $t$ and the angle $\theta$ there are two conserved quantities, the energy $E$ and the angular momentum $J$. From these two conservation laws and from the invariance of the action under arbitrary reparametrizations of the world-line coordinate $u$, one can find a differential equation relating $\theta$ and the radial coordinate

$$
\frac{d \theta}{d r}=-\frac{b}{r^{2} \sqrt{\frac{\beta}{\alpha}-\frac{b^{2}}{r^{2}}}} \Leftrightarrow \frac{d \theta}{d \rho}=\frac{\hat{b}}{\sqrt{1+\rho^{7-p}-\hat{b}^{2} \rho^{2}}},
$$

where $b=J / E, \rho=R_{p} / r, \hat{b}=b / R_{p}$. Notice that the previous result depends only on the ratio $\alpha / \beta$ and it is therefore invariant under an $r$-dependent rescaling of the whole metric. This means that both the string and the Einstein frame metric yield the same equation (4.2) for the classical trajectory. In the last step of (4.2) we used the actual form of $\alpha / \beta$ given in eq. (1.1).

From (4.2) we see that the value of the angle $\theta$ at the turning point $r_{*}$ is

$$
\theta\left(r_{*}\right)=\int_{\infty}^{r_{*}} \frac{d \theta}{d r} d r=\int_{0}^{\rho_{*}} d \rho \frac{\hat{b}}{\sqrt{1+\rho^{7-p}-\hat{b}^{2} \rho^{2}}}
$$

where $\rho_{*}=R_{p} / r_{*}$ is the smallest root of the equation $1+\rho^{7-p}-\hat{b}^{2} \rho^{2}=0$. Since the trajectory of a probe particle in the metric in eq. (4.1) is symmetric around $r_{*}$, the deflection angle $\Theta_{p}$ is given by

$$
\Theta_{p}=2 \theta\left(r_{*}\right)-\pi \Rightarrow \Theta_{p}=2 \int_{0}^{\rho_{*}} d \rho \frac{\hat{b}}{\sqrt{1+\rho^{7-p}-\hat{b}^{2} \rho^{2}}}-\pi .
$$

The integral can be performed explicitly in terms of elementary functions for the cases $p=5,6$ yielding

$$
\tan \frac{\Theta_{6}}{2}=\frac{1}{2 \hat{b}}, \quad \Theta_{5}=\frac{\pi}{\sqrt{1-\left(\frac{1}{\hat{b}}\right)^{2}}}-\pi .
$$

For the case $p=3$ we get instead

$$
\Theta_{3}=2 \sqrt{1+k_{3}^{2}} K\left(k_{3}\right)-\pi, \quad K\left(k_{3}\right)=\int_{0}^{1} \frac{d v}{\sqrt{\left(1-v^{2}\right)\left(1-k_{3}^{2} v^{2}\right)}},
$$


where $K$ is the complete elliptic integral of first kind and

$$
k_{3}=-1+\frac{\hat{b}}{2}\left(\hat{b}-\sqrt{\hat{b}^{2}-4}\right) .
$$

Similar expressions involving elliptic integrals can be given for $p=1$ and $p=4$ while for the cases $p=0$ and $p=2$ we do not have an expression in terms of special functions. Nonetheless, the leading and next-to-leading terms in the large impact parameter expansion for arbitrary $p$ can be computed and read

$$
\Theta_{p}=\sqrt{\pi}\left[\frac{\Gamma\left(\frac{8-p}{2}\right)}{\Gamma\left(\frac{7-p}{2}\right)}\left(\frac{R_{p}}{b}\right)^{7-p}+\frac{1}{2} \frac{\Gamma\left(\frac{15-2 p}{2}\right)}{\Gamma(6-p)}\left(\frac{R_{p}}{b}\right)^{2(7-p)}+\ldots\right],
$$

in perfect agreement with the string calculations in eq. (3.10).

We should make a remark at this point about the order at which we expect to find agreement between the string calculation and the external metric one. In matching the two results we have identified the impact parameter $b$ of the string calculation, defined by the Fourier transform (3.6), with the impact parameter of the geodesic calculation, defined as $b=J / E$. We expect such an identification to fail at order $\Theta_{p}^{3}$, when, for instance, $\sin \Theta_{p}$ starts to differ from $\Theta_{p}$. The analysis of the scattering amplitude in eq. (3.6) can be made more precise by performing a partial wave decomposition and by rewriting the amplitude in terms of the conserved quantities $E$ and $J$. In the case of the high-energy string-string interaction this approach was discussed in $[8,46,47]$ and it should be possible to adapt their derivation to the string-brane scattering analyzed in this paper.

\subsection{Tidal excitation of the closed string at leading order}

We shall now compare the results of the previous section concerning the possible excitation of the probe closed strings with what one obtains by quantizing a closed string in the nontrivial metric (1.1). A similar exercise in the case of string-string collisions leads, to leading order, to agreement with expectations for quantizing a closed string in an Aichelburg-Sexl metric [48-51], see also the discussion in [46, 47].

In the case at hand the end result of the string calculation is the eikonal-operator formula (3.1). Such a formula refers to the leading contribution in $R_{p} / b$ but is supposed to hold at all orders in $\alpha^{\prime} / b^{2}$. On the other hand, the curved-spacetime calculation we shall present below is limited to small string fluctuations around the point-particle null geodesic while, in principle, it can be extended to higher orders in $R_{p} / b$. Our comparison will be made in the overlap of the domains of validity of the two calculations, namely at leading order both in $\alpha^{\prime} / b^{2}$ and in $R_{p} / b$. In spite of this the perfect agreement between the two calculations appears to be almost miraculous, given the very different techniques being used, and represents in our opinion a highly non trivial check of the validity of our approach.

In order to set up the curved space calculation we shall follow [52] and rewrite the full metric as

$$
d s^{2}=\alpha(r)\left(-d t^{2}+\sum_{a=1}^{p}\left(d x^{a}\right)^{2}\right)+\beta(r)\left(d r^{2}+r^{2}\left(d \theta^{2}+\sin ^{2} \theta d \Omega_{7-p}^{2}\right)\right)
$$


where in our case $\beta(r)=1 / \alpha(r)=\sqrt{H(r)}$. This reduces to (4.1) on the plane of the null geodesic considered in section 4.1 and we are now interested in describing the metric around such a geodesic. This is done by first going to a system of adapted coordinates $u, v, z, x^{a}, y^{i}$ in which the geodesic corresponds to constant $v, z, x^{a}, y^{i}$ and $u=u(r)$ plays the role of the affine parameter along the geodesic

$$
\begin{aligned}
d v & =-d t+b d \theta+C d r, & d z & =d(\theta+\bar{\theta}(u)), \\
d u & = \pm \frac{\beta d r}{C}, & C(r) & =\sqrt{\frac{\beta(r)}{\alpha(r)}-\frac{b^{2}}{r^{2}}}
\end{aligned}
$$

Here $\bar{\theta}(u)$ is the angular coordinate $\theta$, evaluated along the null geodesic as in (4.2) and expressed in terms of $u$ via eq. (4.11). The physical meaning of the 7 coordinates $x^{a}, y^{i}$ is that they represent fluctuations orthogonal to the null geodesic and, respectively, parallel to the directions of the brane world-volume $\left(x^{a}\right)$ or along the $(7-p)$ directions $\left(y^{i}\right)$ which are orthogonal both to the brane and to the plane of the geodesic. The $z$ coordinate is orthogonal to the brane, but lies in the plane of the geodesic. In our conventions the point $u=0$ corresponds to the turning point $r_{*}$ and the choice of sign in the equation for $u$ depends on the point of the geodesic we are considering: we choose the minus sign in the approaching region and so parametrize the part of the geodesic from infinity to $r_{*}$ with the interval $-\infty<u \leq 0$; for the remaining part from $r_{*}$ to infinity we choose the plus sign and so it corresponds to the interval $0 \leq u<\infty$. In these adapted coordinates the metric takes the form

$$
d s^{2}=2 d u d v-\alpha d v^{2}+2 b \alpha d v d z+r^{2} \alpha C^{2} d z^{2}+\alpha d x^{a} d x^{a}+\beta r^{2} \sin ^{2}(z-\bar{\theta}) d \Omega_{7-p}^{2} .
$$

At this point we can take the Penrose limit of the above metric (corresponding to the highenergy limit for the probe) and focus on a small neighborhood around the null geodesic by expanding to the quadratic order the dependence on all coordinates transverse to the light-cone and keeping only the linear terms in $v$. This clearly eliminates the $d v^{2}$ and $d v d z$ terms, then by the following change of coordinates

$$
\begin{aligned}
& z=\frac{\hat{y}^{0}}{\sqrt{r^{2} \alpha C^{2}}}, \quad y^{i}=\frac{\hat{y}^{i}}{\sqrt{\beta} r \sin \bar{\theta}}, \quad x^{a}=\frac{\hat{x}^{a}}{\sqrt{\alpha}}, \\
& v=\hat{v}+\frac{1}{2}\left[\sum_{a=1}^{p} \hat{x}_{a}^{2} \partial_{u} \ln (\sqrt{\alpha})+\sum_{i=1}^{7-p} \hat{y}_{i}^{2} \partial_{u} \ln (\sqrt{\beta r \sin \bar{\theta}})+\hat{y}_{0}^{2} \partial_{u} \ln \left(\sqrt{r^{2} \alpha C^{2}}\right)\right]
\end{aligned}
$$

we can bring the metric in the pp-wave form [52]

$$
\begin{aligned}
d s^{2} & =2 d u d \hat{v}+\sum_{a=1}^{p} d \hat{x}_{a}^{2}+\sum_{i=1}^{7-p} d \hat{y}_{i}^{2}+d \hat{y}_{0}^{2}+\mathcal{G}\left(u, \hat{x}^{a}, \hat{y}^{i}, \hat{y}^{0}\right) d u^{2} \\
\mathcal{G} & =\frac{\partial_{u}^{2} \sqrt{\alpha}}{\sqrt{\alpha}} \sum_{a=1}^{p} \hat{x}_{a}^{2}+\frac{\partial_{u}^{2}(\sqrt{\beta} r \sin \bar{\theta})}{\sqrt{\beta} r \sin \bar{\theta}} \sum_{i=1}^{7-p} \hat{y}_{i}^{2}+\frac{\partial_{u}^{2} \sqrt{\beta r^{2}-b^{2} \alpha}}{\sqrt{\beta r^{2}-b^{2} \alpha}} \hat{y}_{0}^{2} \\
& \equiv \mathcal{G}_{x} \sum_{a=1}^{p} \hat{x}_{a}^{2}+\mathcal{G}_{y} \sum_{i=1}^{7-p} \hat{y}_{i}^{2}+\mathcal{G}_{0} \hat{y}_{0}^{2}
\end{aligned}
$$


The bosonic part of the string sigma model then reads

$$
S=S_{0}-\frac{1}{4 \pi \alpha^{\prime}} \int d \tau \int_{0}^{2 \pi} d \sigma \eta^{\alpha \beta} \partial_{\alpha} U \partial_{\beta} U \mathcal{G}\left(U, X^{a}, Y^{i}, Y^{0}\right),
$$

where from now on we will drop the hats on the coordinates. In the previous equation $S_{0}$ is the free Minkowski string action, $\eta_{\alpha \beta}$ the flat worldsheet metric and $\mathcal{G}$ is as in eq. (4.14) but now considered as a function of the string coordinates (denoted by capital letters). In these coordinates string quantization is quite easy if we choose (within the class of orthonormal gauges) the light-cone gauge ${ }^{7}$

$$
U(\sigma, \tau)=\alpha^{\prime} p^{u} \tau \rightarrow \alpha^{\prime} E \tau
$$

This choice drastically simplifies the non-trivial part of the sigma model action to give

$$
\begin{aligned}
S-S_{0} & =\frac{E}{2} \int_{0}^{2 \pi} \frac{d \sigma}{2 \pi} \int_{-\infty}^{+\infty} d u \mathcal{G}\left(u, X^{a}\left(\sigma, u / \alpha^{\prime} E\right), Y^{i}\left(\sigma, u / \alpha^{\prime} E\right), Y^{0}\left(\sigma, u / \alpha^{\prime} E\right)\right) \\
& \rightarrow \frac{E}{2} \int_{0}^{2 \pi} \frac{d \sigma}{2 \pi} \int_{-\infty}^{+\infty} d u\left(\mathcal{G}_{x}(u) \sum_{a=1}^{p} X_{a}^{2}(\sigma, 0)+\mathcal{G}_{y}(u) \sum_{i=1}^{7-p} Y_{i}^{2}(\sigma, 0)+\mathcal{G}_{0}(u) Y_{0}^{2}(\sigma, 0)\right) \\
& \equiv \frac{E}{2} \int_{0}^{2 \pi} \frac{d \sigma}{2 \pi}\left(c_{x} \sum_{a=1}^{p} X_{a}^{2}(\sigma, 0)+c_{y} \sum_{i=1}^{7-p} Y_{i}^{2}(\sigma, 0)+c_{0} Y_{0}^{2}(\sigma, 0)\right)
\end{aligned}
$$

where in the second step we have used the high-energy limit. In this way the integrals over $u$ decouple from the string coordinates and just provide $c$-number coefficients $\mathcal{G}$ to the quadratic action of the fluctuations. Because of the change of sign in $d u / d r$ at $u=0$ the integrals under consideration are twice the same integrals between 0 and $\infty$.

At first sight all the fluctuations of the closed strings, both in the Neumann and in the Dirichlet directions, appear to be excited. It turns out, however, that the former are not excited to the leading order in $R_{p} / b$ to which we are working. This is because we can write

$$
c_{x}=2 \int_{0}^{\infty} \frac{\partial_{u}^{2} \sqrt{\alpha}}{\sqrt{\alpha}} d u=2 \int_{0}^{\infty}\left[\left(\partial_{u} \ln \sqrt{\alpha}\right)^{2}+\partial_{u}^{2}(\ln \sqrt{\alpha})\right] d u .
$$

In the second integral the first term is proportional to $\left(\frac{R_{p}}{b}\right)^{2(7-p)}$ and so it yields a contribution of higher order in $R_{p} / b$, while the second term, being the total derivative of a function that vanishes on the integration boundaries, gives zero (see appendix $\mathrm{C}$ for more details). Hence, to this order, $c_{x}=0$.

This is not true for the other two fluctuations. The coefficient of the $Y_{i}$ fluctuations is quite simple to evaluate by writing, using again the trick that we have just used,

$$
c_{y}=2 \int_{0}^{\infty}\left[\left(\partial_{u} \ln \sqrt{\beta} r \sin \bar{\theta}\right)^{2}+\partial_{u}^{2}(\ln \sqrt{\beta} r \sin \bar{\theta})\right] d u
$$

As in the previous case, the first term does not contribute at the order $\left(\frac{R_{p}}{b}\right)^{7-p}$ we are interested in, but, as shown in appendix $\mathrm{C}$, this time the $u=0$ boundary provides a non-vanishing contribution for the second term and we obtain $c_{y}=-\Theta_{p} / b$.

\footnotetext{
${ }^{7}$ The factor 2 usually present in the r.h.s of eq. (4.16) is absent here because we take $0 \leq \sigma \leq 2 \pi$.
} 
Inserting the leading value of $\Theta_{p}$ from (4.8) we see that the coefficient of $Y^{2}$ agrees with the one obtained in section 3 from the second derivative of the phase shift in the directions orthogonal to the brane and to $\vec{b}$, i.e.

$$
c_{y}=-\frac{\sqrt{\pi}}{b}\left(\frac{R_{p}}{b}\right)^{7-p} \frac{\Gamma\left(\frac{8-p}{2}\right)}{\Gamma\left(\frac{7-p}{2}\right)} \Longrightarrow \frac{E}{2} c_{y}=Q_{\perp}(s, b) .
$$

Computation of the coefficient of the $Y_{0}$ fluctuations is a little more involved since the analogue of the square of the first derivative in (4.18) is not subleading: one has to perform carefully the second derivative, expand it to leading order in $R_{p} / b$, and integrate explicitly each term over $u$. This lengthy but straightforward exercise (reported in appendix C) gives for the coefficient of the $Y_{0}$ fluctuations

$$
c_{0}=\frac{\sqrt{\pi}}{b}(7-p)\left(\frac{R_{p}}{b}\right)^{7-p} \frac{\Gamma\left(\frac{8-p}{2}\right)}{\Gamma\left(\frac{7-p}{2}\right)} \Longrightarrow \frac{E}{2} c_{0}=Q_{\|}(s, b),
$$

again in perfect agreement with the string calculation.

\section{Conclusions}

The eikonal operator derived in this paper allows us to describe different regimes of the scattering of a closed string off a stack of $N$ Dp-branes. These regimes are characterized by the relative magnitude of the scales involved in the dynamics, namely the energy of the colliding string $E$, the impact parameter $b$, the curvature radius $R_{p}$ of the brane background and the string length $l_{s}$. In analogy with ACV, it is useful to draw, as in figure 1 , the different regimes in an $\left(R_{p}, b\right)$ plane marking on both axis the effective string length $l_{s}(s)=l_{s} \sqrt{\ln \alpha^{\prime} s}$. Notice that, in our case, the parameter $R_{p}$, which sets the scale of the effective geometry, is independent of $E$ and we can therefore describe the various regimes at a fixed large energy $\alpha^{\prime} s \gg 1$. We have also limited the diagram to the region $R_{p}, b>l_{s}(s)$ since, in this paper, we have not discussed the new phenomena that occur for $b$ or $R_{p}$ smaller than $l_{s}(s)$.

There are various distinct regimes that we shall now describe by going from the topleft to the bottom-right part of figure 1 . For very large $b$ (above the blue line) the eikonal phase is small and we are in a perturbative - rather than in a classical - regime. This region corresponds to infinitesimal deflection angles (as $b$ becomes larger and larger). The straight black lines at 45 degrees represent curves of constant deflection angle: the solid one is meant to represent a critical ratio of $R_{p} / b$ below which, classically, a test point particle is captured, while the dashed one represents a typical, small deflection angle $\Theta \ll 1$. The red line represents instead the impact parameter $b_{D}$, defined in eq. (3.26), below which tidal excitation becomes relevant.

In the region bordered by the blue, the red and the black-dashed line the leading eikonal approximation is reliable and elastic unitarity holds to a very good approximation. Below the red and above the black-dashed line we have the tidal excitations computed in section 2.3, while in the complementary region (above the red but below the black-dashed line) we need to use an improved eikonal including higher-order classical corrections whose 


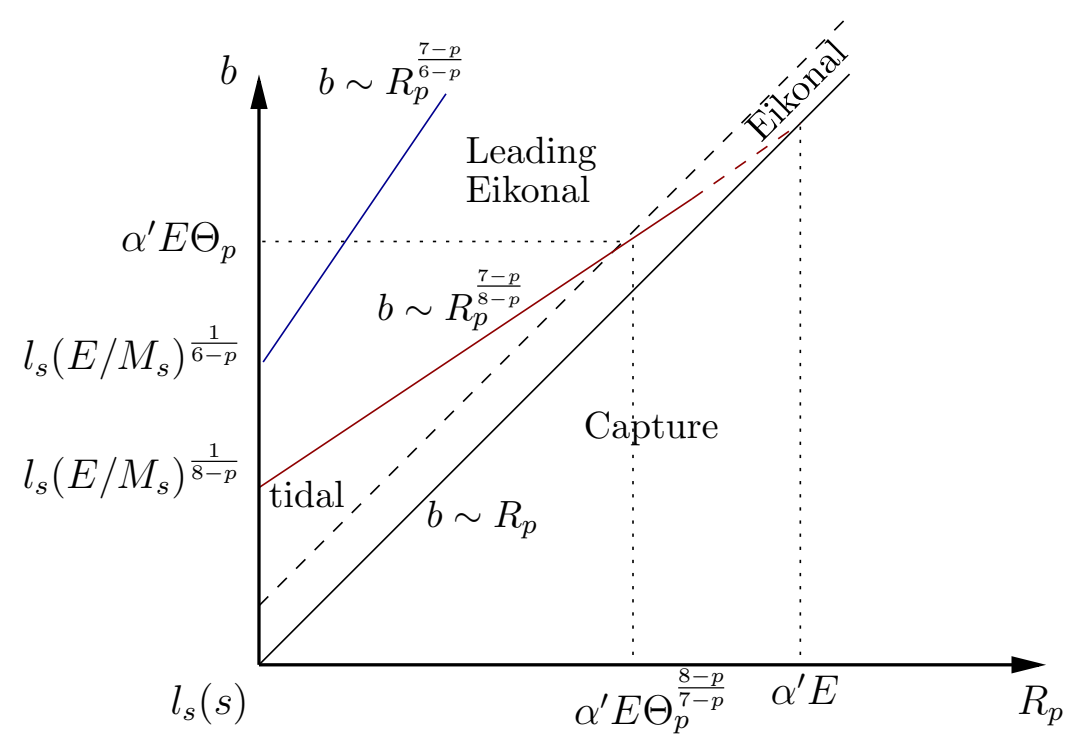

Figure 1. Various qualitative regimes in the scattering of a closed string of fixed high energy off a stack of Dp-branes as a function, in a $\log -\log$ plot, of $R_{p}$ and $b$, both taken to be bigger than the effective string scale $l_{s}(s)$. The different regions are discussed in the text.

first term was computed in section 2.2. Tidal effects, however, should be small in this region. Clearly, as we go further down in the diagram, we encounter a region in which both kinds of correction (string-size and classical) come together. When the impact parameter is close to $R_{p}$, we expect large corrections to the eikonal operator discussed in this paper and, for this reason, the solid line, signaling the onset of the string-tidal effects, ends before meeting the $b \sim R_{p}$ line.

In this paper we have focused on the upper part of the diagram in figure 1 , where the impact parameter is large compared to $R_{p}$ and gravity effects dominate the interaction between the Dp-branes and the string probe. It is in this region that the comparison between the string dynamics in the extremal p-brane background and the dynamics resulting from the string scattering amplitudes is most transparent. Having tested our approach in this regime, there are two other interesting but more difficult regions to consider. The first is the string region $R_{p}<l_{s}(s)$, where string corrections to the geometry are important and one expects that the dynamics will be very different from the one predicted by the effective background in eqs. (1.1), (1.2). The second is the region $b \sim R_{p}>l_{s}(s)$, where the dynamics should be dominated by strong gravity effects. As we lower the impact parameter to study these two new regions, we should be able to make contact with the analysis of high-energy amplitudes at fixed angle as discussed in [9] and, in the context of D-branes, in $[53,54]$. Moreover, in these regimes the decomposition of the amplitude in partial waves should provide a very useful tool to clarify the main features of the scattering process, as discussed in $[8,46,47]$.

The quantitative analysis of a string-brane scattering process in the string region $R_{p}<$ $l_{s}(s)$ and in the strong gravity region $b \sim R_{p}$ requires some control on both the classical and string corrections to the leading eikonal operator. An example of the possible effects 
of these corrections is provided by the string excitations along the Dp-brane world-volume. Although these excitations are absent in (2.40), the analysis of section 4 suggests that they should become relevant at smaller impact parameters. An effective way of studying these kinds of corrections is to calculate amplitudes similar to those discussed in this paper but involving also massive string states [55]. From a more general perspective, it would be very interesting to derive and interpret the corrections to the eikonal operator which

are of a higher order in $\frac{R_{p}}{b}$ and $\frac{l_{s}(s)}{b}$ by computing explicitly the high-energy behaviour of amplitudes with three or more boundaries. This kind of analysis might be possible by using Regge-Gribov techniques [4, 5].

Another interesting generalization of our setup is to change the nature of the massive target and possibly also the nature of the asymptotic space by including compact directions. For instance, bound states of different D-branes, such as the D1/D5 system, have been studied intensively in recent years and provide a tractable system that has an exponential degeneracy of states (see for instance [56] and references therein). The geometries corresponding to these states are known and have some interesting features such as the breaking of rotational symmetry in the transverse space. It would be interesting to generalize the string computations of $[57,58]$ and to see how the properties of these microstate solutions affect the high-energy scattering considered in this paper. Furthermore, by adding a momentum charge to the D1/D5 bound state, one obtains the configurations studied in [59] which are related to a black hole with a macroscopic horizon.

In all these examples the most interesting region from the point of view of gravity and black-hole physics is that of small impact parameters $\left(b \lesssim R_{p}\right)$, where, as previously noted, the eikonal phase receives large classical corrections. However, as was the case for the simpler regime analysed in this paper, the mixed open/closed string amplitudes which describe the collision of a string with a D-brane configuration might be more tractable at high energies. They could then provide an ideal framework for discussing in a quantitative way the dynamics of matter falling beyond a horizon and the resulting small perturbations of a black hole above its ground state.

\section{Acknowledgments}

We would like to thank Daniele Amati, Costas Bachas, William Black, Marcello Ciafaloni, Emilian Dudas, Stefano Giusto, Vishnu Jejjala, Lello Marotta, Cristina Monni, Yaron $\mathrm{Oz}$ and Gabriele Travaglini for fruitful discussions. This work is partially supported by INFN and by STFC under the Rolling Grant ST/G000565/1. GD would like to thank the members of the Laboratoire de Physique Théorique de l'Ecole Normale Supérieure in Paris, where part of this work was done, for the warm hospitality extended to him.

\section{A The saddle point in the annulus moduli space}

The string amplitude (2.14) contains an integral over the moduli space of a cylinder with two punctures in its interior representing the external closed string states. Our parametrization of this surface is summarized in figure 2 . 


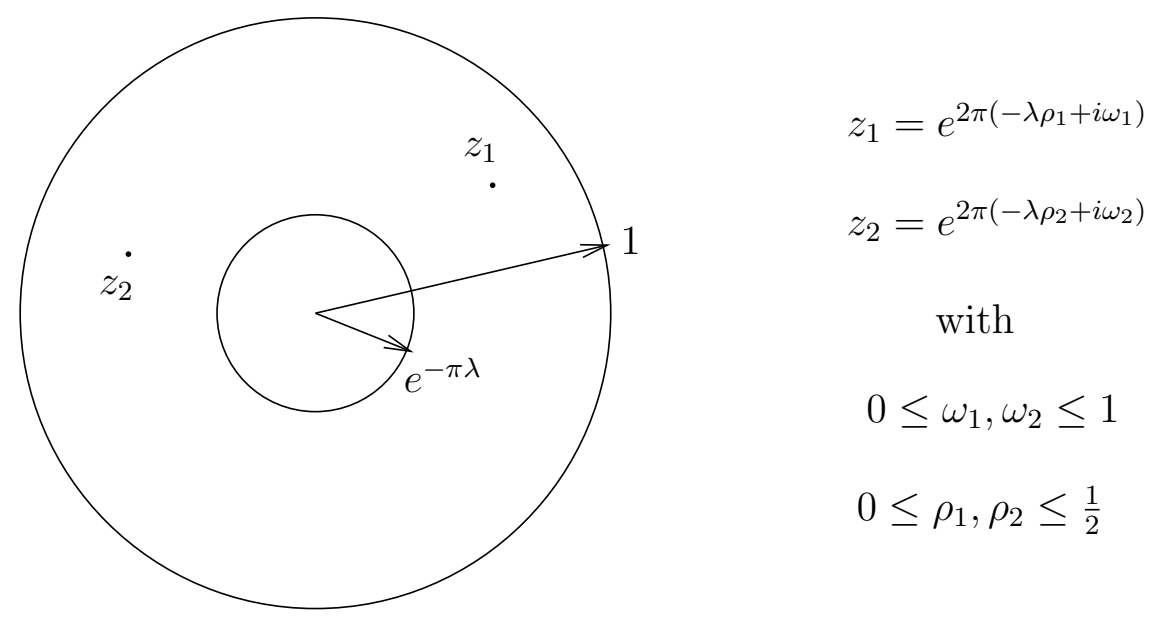

Figure 2. The string world-sheet is represented by the annulus between the outer circle of radius 1 and the inner circle of radius $e^{-\pi \lambda}$. The two external states are represented by the two punctures located at $z_{1}$ and $z_{2}$.

We are interested in a particular kinematics where both the impact parameter and the energy of the incident states are much larger than the scale fixed by the string length: $\sqrt{\alpha^{\prime}} E \gg 1$ and $b \gg l_{s}(s)$. In this regime the integral over the world-sheet moduli is dominated by the contribution of a critical region at small values of $\rho=\rho_{1}-\rho_{2}$ and large values of $\lambda$. Indeed, the large $b$ limit implies that the momentum exchanged between the string state and the D-branes is small and therefore the corner of the string integration region that is more relevant is $\lambda \rightarrow \infty$. Moreover, we are taking the high-energy limit for the external states and, as we will see, the integrand is exponentially suppressed unless $\rho \sim 0$.

The Jacobi theta function

$$
\theta_{1}(\nu \mid \tau)=-2 e^{\pi i \tau / 4} \sin (\pi \nu) \prod_{m=1}^{\infty}\left[\left(1-e^{2 \pi i m \tau}\right)\left(1-2 \cos (2 \pi \nu) e^{2 \pi i m \tau}+e^{4 \pi i m \tau}\right)\right]
$$

which appears in the definitions (2.16) and (2.17) has a simple expansion in our critical region. We can then derive the following expressions for the large- $\lambda$ behaviour of $V_{s}$ and $V_{t}$

$$
\begin{aligned}
V_{s} & \sim-2 \pi \lambda \rho^{2}-4\left(\sin ^{2} \pi \omega+\sinh ^{2} \pi \lambda \rho\right)\left(e^{-2 \pi \lambda \zeta}+e^{-2 \pi \lambda(1-\zeta)}\right), \\
V_{t} & \sim-2 \pi \lambda\left[\zeta(1-\zeta)+\rho^{2}\right]+\ln \left[4 \sin ^{2} \pi \omega+4 \sinh ^{2} \pi \lambda \rho\right] .
\end{aligned}
$$

In the expansion for $V_{t}$ we kept only the contributions that do not have any exponential factors, while for $V_{s}$ we also included the exponential terms, since they are sizable as soon as $\lambda>\ln \alpha^{\prime} s$. The first term in each expression corresponds to the leading field theory contribution considered in section 2.2, while the other terms yield the sub-leading field theory contributions and the string corrections.

Let us now focus on the integral in the square parenthesis of (2.14). The integrand does not depend on $\sigma=\omega_{1}+\omega_{2}$ and is periodic under the shift $\omega \rightarrow \omega+1$, so we can perform the integral over $\sigma$ and obtain

$$
I=\int_{0}^{\infty} d \lambda \int_{0}^{1} d \zeta \int_{-\zeta}^{\zeta} d \rho \int_{0}^{1} d \omega \lambda^{\frac{p-5}{2}} \mathcal{I}
$$


Since the integrand is an even function of $\rho$, we can restrict the integral over $\rho$ to the interval from 0 to $\zeta$. The leading contribution in the large $E$ limit scales as $E^{3}$ and comes from the region where $\rho$ is set to zero, except for the first term of $V_{s}$, which has a different structure and has to be kept exact. The subleading contribution is related to the expansion of $\mathcal{I}$ at the quadratic order around $\rho=0$ and we will see that it scales as $E$, i.e. with two power less of energy with respect to the leading contribution. Higher orders in the $\rho$ expansion are further suppressed in the large $E$ limit. So we can further approximate the building blocks (A.2) of the integrand and write $\mathcal{I} \sim \mathcal{I}_{l} \mathcal{I}_{s}$ with

$$
\begin{aligned}
& \mathcal{I}_{l}(\rho) \sim e^{2 \pi \lambda \alpha^{\prime} s \rho^{2}+2 \pi \lambda \zeta(1-\zeta) \frac{\alpha^{\prime} t}{4}}, \\
& \mathcal{I}_{s}(\rho) \sim\left(4 \sin ^{2} \pi \omega+4 \pi^{2} \lambda^{2} \rho^{2}\right)^{-\frac{\alpha^{\prime} t}{4}} e^{4 \alpha^{\prime} s\left(\sin ^{2} \pi \omega+\pi^{2} \lambda^{2} \rho^{2}\right)\left(e^{-2 \pi \lambda \zeta}+e^{-2 \pi \lambda(1-\zeta)}\right),}
\end{aligned}
$$

where we wrote explicitly only the dependence on $\rho$. We then separate the two contributions mentioned above by writing $I=I_{1}+I_{2}$

$$
\begin{aligned}
& I_{1}=\int_{0}^{\infty} d \lambda \int_{0}^{1} d \zeta \int_{\rho \sim 0} d \rho \int_{0}^{1} d \omega \mathcal{I}_{l}(\rho) \mathcal{I}_{s}(\rho=0), \\
& I_{2}=\int_{0}^{\infty} d \lambda \int_{0}^{1} d \zeta \int_{\rho \sim 0} d \rho \int_{0}^{1} d \omega \mathcal{I}_{l}(\rho)\left[\mathcal{I}_{s}(\rho)-\mathcal{I}_{s}(\rho=0)\right] .
\end{aligned}
$$

Let us first describe how to evaluate the leading term $I_{1}$, following closely $[1,2]$. We can regularize the integral over $\rho$ by performing a Wick rotation on the energy of the external states $E \rightarrow i E_{e}$. Then we perform the saddle point integration over $\rho$ and, after Wick rotating back to Minkowskian energy, we obtain

$$
I_{1}=\frac{i}{\sqrt{2 \alpha^{\prime} s}} \int_{0}^{\infty} d \lambda \int_{0}^{1} d \zeta \int_{0}^{1} d \omega \lambda^{\frac{p}{2}-3} e^{2 \pi \lambda \zeta(1-\zeta) \frac{\alpha^{\prime} t}{4}} \mathcal{I}_{s}(\rho=0) .
$$

In order to deal with the integral over $\omega$, we expand the exponential in $\mathcal{I}_{s}(\rho=0)$ as follows

$$
e^{4 \alpha^{\prime} s \sin ^{2} \pi \lambda \omega\left(e^{-2 \pi \lambda \zeta}+e^{-2 \pi \lambda(1-\zeta)}\right)}=\sum_{n=0}^{\infty} \sum_{m=0}^{\infty} \frac{1}{n ! m !}\left(4 \alpha^{\prime} s \sin ^{2} \pi \omega\right)^{n+m} e^{-2 n \pi \lambda \zeta} e^{-2 m \pi \lambda(1-\zeta)},
$$

and perform the integral by setting $x=\pi \omega$ and using

$$
B(a, b)=2 \int_{0}^{\pi / 2} d x \sin ^{2 a-1} x \cos ^{2 b-1} x .
$$

The integral over $\lambda$ can then be evaluated using the integral representation of the gamma function and the result reads

$$
I_{1}=i \frac{4^{-\frac{\alpha^{\prime} t}{4}}}{\sqrt{2 \alpha^{\prime} s}} \sum_{n, m=0}^{\infty} \int_{0}^{1} d \zeta \frac{1}{n ! m !} \frac{\left(4 \alpha^{\prime} s\right)^{n+m} \Gamma\left(\frac{p-4}{2}\right) B\left(\frac{1}{2}+n+m-\frac{\alpha^{\prime} t}{4}, \frac{1}{2}\right)}{\pi^{\frac{p-4}{2}+1}\left[2 n \zeta+2 m(1-\zeta)-\zeta(1-\zeta) \frac{\alpha^{\prime} t}{2}\right]^{\frac{p-4}{2}}} .
$$

In this equation $\zeta$ plays the role of a Feynman parameter for a diagram with two propagators and an integrated momentum over $8-p$ dimensions

$$
\int_{0}^{1} d \zeta \frac{\left(2 \pi \alpha^{\prime}\right)^{\frac{p-8}{2}} \Gamma\left(\frac{p-4}{2}\right)}{\left[2 n \zeta+2 m(1-\zeta)-\zeta(1-\zeta) \frac{\alpha^{\prime} t}{2}\right]^{\frac{p-4}{2}}}=\int \frac{d^{8-p} \mathbf{k}}{(2 \pi)^{8-p}} \frac{1}{\left[2 n+\frac{\alpha^{\prime}}{2} \mathbf{k}^{2}\right]\left[2 m+\frac{\alpha^{\prime}}{2}(\mathbf{k}-\mathbf{q})^{2}\right]}
$$


where $\mathbf{q}$ is a $(8-p)$-dimensional vector whose norm is $\mathbf{q}^{2}=-t$. In terms of this momentum integral, the contribution $I_{1}$ becomes

$$
I_{1}=i \frac{\pi^{1-\frac{p}{2}} 4^{-\frac{\alpha^{\prime}}{4} t}}{\sqrt{2 \alpha^{\prime} s}\left(2 \pi \alpha^{\prime}\right)^{\frac{p}{2}-4}} \sum_{n, m=0}^{\infty} \frac{\left(4 \alpha^{\prime} s\right)^{n+m}}{n ! m !} \int \frac{d^{8-p} \mathbf{k}}{(2 \pi)^{8-p}} \frac{B\left(\frac{1}{2}+n+m-\frac{\alpha^{\prime} t}{4}, \frac{1}{2}\right)}{\left[2 n+\frac{\alpha^{\prime}}{2} \mathbf{k}^{2}\right]\left[2 m+\frac{\alpha^{\prime}}{2}(\mathbf{k}-\mathbf{q})^{2}\right]} .
$$

We can now transform the sums over $n$ and $m$ into two integrals over the complex plane by using

$$
\sum_{m=0}^{\infty} \frac{1}{m !} \frac{f(m) s^{m}}{m+t}=-\int_{\mathcal{C}} \frac{d z_{m}}{2 \pi i} \frac{f\left(z_{m}\right) s_{m}^{z}}{z_{m}+t} e^{-i \pi z_{m}} \Gamma\left(-z_{m}\right)
$$

and a similar expression for the sum over $n$. The contour $\mathcal{C}$ runs anti-clockwise around the real positive axis in the complex plane (clearly this identity holds if $f$ does not have any pole on the real positive axis). Deforming the integration over the two complex variables $z_{n}, z_{m}$, we pick the contributions of the poles on the negative real axis

$$
\begin{aligned}
I_{1} \sim & i \frac{\pi^{1-\frac{p}{2}}\left(e^{-i \pi} \alpha^{\prime} s\right)^{\frac{\alpha^{\prime}}{4} t}}{\sqrt{2 \alpha^{\prime} s}\left(2 \pi \alpha^{\prime}\right)^{\frac{p}{2}-4}} \int \frac{d^{8-p} \mathbf{k}}{(2 \pi)^{8-p}}\left\{-\Gamma\left[\frac{1}{2}-\frac{\alpha^{\prime}}{4} t\right] \frac{\left(4 e^{-i \pi} \alpha^{\prime} s\right)^{-\frac{1}{2}}}{\left[\alpha^{\prime}(\mathbf{k}-\mathbf{q})^{2}\right]\left[\frac{1}{2}-\frac{\alpha^{\prime}}{4}\left(t+\mathbf{k}^{2}\right)\right]}\right. \\
& +\Gamma\left[\frac{\alpha^{\prime}}{4} \mathbf{k}^{2}\right] \Gamma\left[\frac{1}{2}-\frac{\alpha^{\prime}}{4}\left(t+\mathbf{k}^{2}\right)\right] \frac{\left(4 e^{-i \pi} \alpha^{\prime} s\right)^{-\frac{1}{2}}}{\alpha^{\prime}\left(\mathbf{k}^{2}+(\mathbf{k}-\mathbf{q})^{2}+t\right)-2} \\
& \left.+\frac{1}{4} \Gamma\left[\frac{\alpha^{\prime}}{4} \mathbf{k}^{2}\right] \Gamma\left[\frac{\alpha^{\prime}}{4}(\mathbf{q}-\mathbf{k})^{2}\right] B\left(\frac{1}{2}+\frac{\alpha^{\prime}}{2}\left(-\mathbf{k}^{2}+\mathbf{k} \cdot \mathbf{q}\right), \frac{1}{2}\right)\left(4 e^{-i \pi} \alpha^{\prime} s\right)^{\frac{\alpha^{\prime}}{2}\left(-\mathbf{k}^{2}+\mathbf{k} \cdot \mathbf{q}\right)}\right\},
\end{aligned}
$$

where we included only the first pole in the Euler beta function in (A.13), since the remaining poles (in $z_{n}+z_{m}+1 / 2-\alpha^{\prime} t / 4=-k$ with $k \geq 1$ ) yield contributions that are finite or small in the high-energy limit. Each line in this equation contains some non-physical poles when $\mathbf{k}$ takes particular values. For instance, the first term of the integral is divergent when $2-\alpha^{\prime}\left(t+\mathbf{k}^{2}\right)=0$, but this singularity is cancelled by a similar pole in the second line of (A.15). Similarly the first pole of the beta function in the last line is cancelled by the other pole of the second term (while the remaining poles of the beta function would be cancelled by the terms we neglected in (A.15)). By including the prefactor in (2.14) and using the duplication formula $\Gamma(x) \Gamma\left(x+\frac{1}{2}\right)=\sqrt{\pi} 2^{1-2 x} \Gamma(2 x)$, we can finally rewrite the last term of (A.15) as in (2.33).

The Euler beta function in the last line of (A.15) can be written in terms of the expectation value of two tachyon-like string vertices $[1,2]$

$$
\left\langle 0\left|\prod_{i=1}^{2} \int_{0}^{2 \pi} \frac{d \sigma_{i}}{2 \pi}: e^{i \mathbf{k}_{i} \hat{X}\left(\sigma_{i}\right)}:\right| 0\right\rangle=\frac{2^{\alpha^{\prime} \mathbf{k}_{1} \mathbf{k}_{2}}}{\pi} B\left(\frac{1}{2}+\frac{\alpha^{\prime}}{2} \mathbf{k}_{1} \mathbf{k}_{2}, \frac{1}{2}\right)
$$

where $\hat{X}(z)$ has the standard mode expansion, but without the zero-modes describing the centre of mass position and momentum

$$
\hat{X}\left(\sigma_{i}\right)=i \sqrt{\frac{\alpha^{\prime}}{2}} \sum_{n \neq 0}\left(\frac{\alpha_{n}}{n} e^{i n \sigma}+\frac{\bar{\alpha}_{n}}{n} e^{-i n \sigma}\right),
$$


which yields the $\operatorname{OPE} \hat{X}(z) \hat{X}(w) \sim-\alpha^{\prime} \ln |1-w / z|$. At this point one can simply follow the same steps as in section 2.3 and use (A.16) with $\mathbf{k}_{1}=\mathbf{k}$ and $\mathbf{k}_{2}=\mathbf{q}-\mathbf{k}$ in order to show that $\mathcal{A}_{2}^{(3)}$, which follows from the last line of (A.15), can be written as the convolution in eq. (2.38).

Let us briefly comment on the leading string corrections at small $\alpha^{\prime} t$ (or at large impact parameters $b$ ), which are contained in the last line of (A.15). They are obtained by first expanding for small $\alpha^{\prime} t$ the r.h.s. of (A.16), or equivalently the vertex $V_{2}$ in (2.34),

$$
V_{2}(\mathbf{k}, \mathbf{q}-\mathbf{k}) \sim 1+\frac{\pi^{2}}{6}\left(\frac{\alpha^{\prime}}{2} \mathbf{k} \cdot(\mathbf{q}-\mathbf{k})\right)^{2}+\ldots
$$

and then by using this approximate form in (2.33). One can then see explicitly that also the contribution of these first string corrections takes the form of a convolution involving the second derivatives of $\mathcal{A}_{1}$ as in (3.11). After rewriting $\mathcal{A}_{2}^{(3)}$ in impact-parameter space, we can compare this result with the expansion of (3.21) for small $\alpha^{\prime} Q_{\perp}$ and small $\alpha^{\prime} Q_{\|}$finding agreement at the quadratic order. The result in (3.21) shows how these string corrections are properly incorporated in the eikonal operator once diagrams with an arbitrary number of boundaries are resummed.

We now analyse the subleading contribution $I_{2}$. For simplicity we shall focus on the term that is non-vanishing in the field theory limit, that is the first term in the series expansion of the exponential in the definition of $\mathcal{I}_{s}$ in (A.5). The other terms can be evaluated in a similar way.

With the change of variable $\sin ^{2} \pi \omega \rightarrow x$, one can see that the integral over $\omega$ yields a hypergeometric function

$$
\begin{aligned}
\int_{0}^{1} d \omega\left(\sin ^{2} \pi \omega+y^{2}\right)^{c} & =y_{2}^{2 c}{ }_{2} F_{1}\left(-c, 1 / 2 ; 1 ;-y^{-2}\right) \\
& =\frac{1}{\sqrt{\pi}} \frac{\Gamma\left(\frac{1}{2}+c\right)}{\Gamma(1+c)}+\frac{y^{1+2 c}}{\sqrt{\pi}} \frac{\Gamma\left(-\frac{1}{2}-c\right)}{\Gamma(-c)}+\ldots
\end{aligned}
$$

where in the second line we wrote the first two terms in the small-y expansion. The first term in (A.19) is cancelled by the contribution of $\mathcal{I}_{s}(0)$ in the definition (A.7) and thus we obtain

$$
I_{2} \sim \frac{\Gamma\left(-\frac{1}{2}+\frac{\alpha^{\prime}}{4} t\right)}{2 \sqrt{\pi} \Gamma\left(\frac{\alpha^{\prime}}{4} t\right)} \int_{0}^{\infty} \frac{d \lambda}{\lambda^{\frac{5-p}{2}}} \int_{0}^{1} d \zeta \int_{\rho \sim 0} d \rho(2 \pi \lambda \rho)^{1-\frac{\alpha^{\prime}}{2} t} e^{2 \pi \lambda \alpha^{\prime}\left(s+\frac{t}{4}\right) \rho^{2}+2 \pi \lambda \zeta(1-\zeta) \frac{\alpha^{\prime} t}{4}} .
$$

The expansion in the second line of (A.19) is appropriate for the regime of very high energies at large, but fixed, values of the impact parameter; in this way $\rho \lambda$, which is of the order of $b /\left(\alpha^{\prime} E\right)$, is small. If we keep increasing $b$ and keep $E$ fixed, we reach a point where $y$ in (A.19) is of order one and so it is more appropriate to expand the result (A.19) for small $c=-\alpha^{\prime} t / 4$ at arbitrary values of $y$. By using for instance appendix B.1 of [60], we obtain

$$
y^{2 c}{ }_{2} F_{1}\left(-c, 1 / 2 ; 1 ;-y^{-2}\right)=1-2 c \ln 2+2 c \pi \lambda \rho+O\left(c^{2}\right),
$$


where in this case we used the full expression for $y=\sinh (\pi \lambda \rho)$, since $\lambda \rho$ is not necessarily small. The $\rho$-independent terms coincide with the small $c$ expansion of the first term in the second line of (A.19) and so they have been already included in $I_{1}$. The term proportional to $\pi \lambda \rho$ in (A.21) agrees with the small $c$ expansion of the second term in (A.19) (remember that in that equation $y$ is approximated with $\pi \lambda \rho)$. This shows that the first order in $t$ of eq. (A.20) captures correctly the contribution of $I_{2}$ also at very large values of the impact parameter $b \geq \alpha^{\prime} E$.

At high energies, the integral over $\rho$ in eq. (A.20) is dominated by the saddle point $\rho \sim 0$ and after a Wick rotation $E \rightarrow i E_{e}$ we obtain the following asymptotic behaviour for $I_{2}$ in the $s \gg t$ limit

$$
I_{2} \sim-\left(\alpha^{\prime} s\right)^{-1+\frac{\alpha^{\prime}}{4} t} e^{-i \pi \frac{\alpha^{\prime}}{4} t} \frac{\Gamma\left(1-\frac{\alpha^{\prime}}{4} t\right) \Gamma\left(-\frac{1}{2}+\frac{\alpha^{\prime}}{4} t\right)}{2 \sqrt{\pi} \Gamma\left(\frac{\alpha^{\prime}}{4} t\right)} \int_{0}^{\infty} \frac{d \lambda}{\lambda^{\frac{5-p}{2}}} \int_{0}^{1} d \zeta\left[2 \pi \lambda e^{-2 \pi \lambda \zeta(1-\zeta)}\right]^{-\frac{\alpha^{\prime}}{4} t} .
$$

The higher powers of $\rho$ in the expansion of the hypergeometric function in (A.19), which we have neglected, would yield contributions that are suppressed by further powers of $1 / E$. This is a consequence of the relation between the expansion in powers of $\rho$ and the asymptotic dependence on the energy mentioned after eq. (A.3). The integrals over $\zeta$ and $\lambda$ are straightforward and yield

$$
I_{2} \sim\left(\alpha^{\prime} s\right)^{-1+\frac{\alpha^{\prime}}{4} t} \frac{\Gamma\left(1-\frac{\alpha^{\prime}}{4} t\right) \Gamma\left(-\frac{1}{2}+\frac{\alpha^{\prime}}{4} t\right)}{2^{\frac{p-1}{2}} \pi^{\frac{p}{2}-1} \Gamma\left(1+\frac{\alpha^{\prime}}{4} t\right)} \frac{B\left(\frac{5-p}{2}+\frac{\alpha^{\prime}}{4} t, \frac{5-p}{2}+\frac{\alpha^{\prime}}{4} t\right)}{\left(-\frac{\alpha^{\prime}}{4} t\right)^{\frac{p-5}{2}-\frac{\alpha^{\prime}}{4} t}} \Gamma\left(\frac{p-3}{2}-\frac{\alpha^{\prime}}{4} t\right) .
$$

From this equation, including the prefactor in (2.14) and sending $\alpha^{\prime} \rightarrow 0$, one can derive eq. (2.31).

\section{B Dilaton scattering in field theory}

In this appendix we provide some details about the field theory diagrams that contribute to the dilaton scattering from a stack of Dp-branes. As in the rest of this paper, we are interested in the high-energy limit of the full scattering amplitude and so we can focus on a subclass of diagrams. At the first order in $\left(R_{p} / b\right)^{7-p}$ there is just a single diagram with a graviton exchange between the probe dilaton and the Dp-branes. It is straightforward to check that the Feynman rules summarized in figure 3 yield the high-energy result of eq. (2.18).

We can use the same Feynman rules to compute the diagrams in figure 4 . They scale as $\left(R_{p} / b\right)^{2(7-p)}$ and should therefore contribute both to the exponentiation of the leading term, see eq. (2.22), and to the subleading term, see eq. (2.31). We also need to consider a second class of diagrams, depicted in figure 5, which are of the same order in $R_{p} / b$ and involve the exchange of gravitons, dilatons and RR-fields. While diagram 4(a) gives both leading $\left(\sim E^{2}\right)$ and sub-leading $(\sim E)$ contributions to the S-matrix, diagram $4(\mathrm{~b})$ and the three diagrams in figure 5 give only sub-leading contributions. It is interesting to note that 


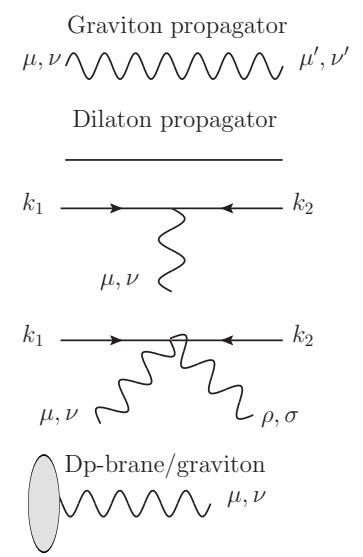

$$
\begin{gathered}
\left(\eta^{\mu \mu^{\prime}} \eta^{\nu \nu^{\prime}}+\eta^{\mu \nu^{\prime}} \eta^{\nu \mu^{\prime}}-\frac{1}{4} \eta^{\mu \nu} \eta^{\mu^{\prime} \nu^{\prime}}\right) \frac{-i}{k^{2}} \\
\frac{-i}{k^{2}} \\
-2 i \kappa\left[k_{1(\mu} k_{2 \nu)}-\frac{1}{2} k_{1 \rho} k_{2}^{\rho} \eta_{\mu \nu}\right] \\
i(2 \kappa)^{2}\left[2 k_{1(\mu} k_{2(\rho} \eta_{\nu) \sigma)}-\frac{1}{2}\left(k_{1(\mu} k_{2 \nu)} \eta_{\rho \sigma}+k_{1(\rho} k_{2 \sigma)} \eta_{\mu \nu}\right)\right]+\ldots \\
-i T_{p} \eta_{\mu \nu}^{\|}=-i \frac{2 R_{p}^{7-p} \frac{9-p}{2}}{\kappa \Gamma\left(\frac{7-p}{2}\right)} \eta_{\mu \nu}^{\|}
\end{gathered}
$$

Figure 3. Here we collect the Feynman rules necessary for the derivation of the diagrams in figure 4 . For the graviton propagator we use the De Donder gauge. As usual, the round parentheses imply a symmetrization of the enclosed indices: for instance we have $k_{1(\mu} k_{2 \nu)}=\left(k_{1 \mu} k_{2 \nu}+k_{1 \nu} k_{2 \mu}\right) / 2$. The symbol $\eta_{\mu \nu}^{\|}$means that the Lorentz indices are restricted to the $p+1$ directions along the brane world-volume. The dots in the quartic vertex stands for terms that are proportional to $k_{1} \cdot k_{2}$ which cannot give contributions growing with $E$ in the diagrams we are interested in.
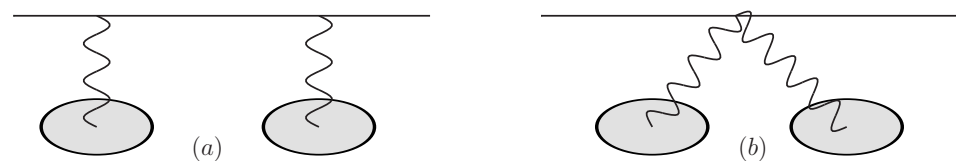

Figure 4. Diagram (a) contributes both to the leading $\left(\sim E^{2}\right)$ and to the subleading $(\sim E)$ terms in the S-matrix. Diagram (b) yields contributions scaling at most as $E$.
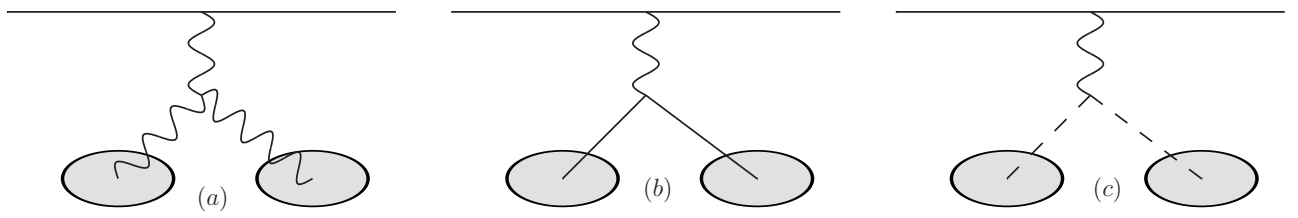

Figure 5. The contributions of these diagrams to the S-matrix scale at most as $E$. The third diagram involves the exchange of a RR-field represented by the dashed lines. At high energies they combine and cancel exactly the leading term of diagram 4(b).

diagram 4(b) cancels, in the high energy limit and in the De Donder gauge, the diagrams in figure 5. As a result, both the leading and the sub-leading contributions to the S-matrix come from diagram 4(a) alone.

Let us start our analysis from diagram 4(a); by using the Feynman rules we have

$$
4(\mathrm{a})=i\left(\kappa T_{p}\right)^{2}(2 \pi)^{p+1} \delta^{(p+1)}\left(p_{1}+p_{2}\right)\left[\int \frac{d^{9-p} k}{(2 \pi)^{9-p}} \frac{4 E^{4}}{\left(p_{1}-k\right)^{2} k^{2}\left(p_{2}+k\right)^{2}}\right],
$$

where $p_{1}, p_{2}$ are the momenta of the two external states and $k$ is the momentum flowing in the dilaton propagator. Notice that the combinations $\left(p_{1}-k\right)$ and $\left(p_{2}+k\right)$ are 
non-trivial only in the $(9-p)$ transverse directions, while $k$ has also a time component and so $k^{2}=-E^{2}+k_{\perp}^{2}$. By performing the same Wick rotation $\left(E=i E_{e}\right)$ used in the string computation, we get standard Euclidean propagators and can introduce Schwinger parameters for evaluating (B.1). By focusing on the square parenthesis we have

$$
[\ldots]=\frac{4 E_{e}^{4}}{(4 \pi)^{\frac{9-p}{2}}} \int_{0}^{\infty} d T T^{-\frac{9-p}{2}+2} \int_{0}^{1} d x \int_{0}^{x} d y \mathrm{e}^{-E_{e}^{2} T y^{2}} \mathrm{e}^{-|t| T(1-x)(x-y)} .
$$

In the high-energy limit, this integral is dominated by the saddle point $y \sim 0$. This is the same pattern seen in the string computation of appendix A and indeed $y$ plays the same role as $\rho$. In order to derive the leading term, one can neglect $y$ in the second exponential and treat the integral over $y$ in the Gaussian approximation. By inserting the result in (B.1) and using (2.6) we obtain the full result (2.22) for the dilaton amplitude at the next-to-leading order in the energy.

The subleading contribution is obtained by expanding to linear order in $y$ the second exponential in (B.2). Again by focusing on the saddle point $y \sim 0$ all integrals can be performed and we obtain the contribution from diagram $4\left(\right.$ a) to $\mathcal{A}_{2}^{(2)}$

$$
R_{p}^{2(7-p)} E^{2} \pi^{\frac{9-p}{2}} 2^{p-6}|t|^{\frac{5-p}{2}} \frac{\Gamma\left(\frac{p-5}{2}\right)}{\Gamma(6-p)},
$$

which agrees with the amplitude $\mathcal{A}_{2}^{(2)}$ of (2.31).

Diagram 4(b) yields

$$
\begin{aligned}
4(\mathrm{~b}) & =\frac{i}{2}\left(\kappa T_{p}\right)^{2}(2 \pi)^{p+1} \delta^{(p+1)}\left(p_{1}+p_{2}\right)\left[\int \frac{d^{9-p} q}{(2 \pi)^{9-p}} \frac{E^{2}(7-p)}{q^{2}\left(q-\left(p_{1}+p_{2}\right)\right)^{2}}\right]+\ldots \\
& =\left(i(2 \pi)^{p+1} \delta^{(p+1)}\left(p_{1}+p_{2}\right)\right) R_{p}^{2(7-p)} E^{2} \pi^{\frac{9-p}{2}} 2^{p-6}|t|^{\frac{5-p}{2}} \frac{\Gamma\left(\frac{p-5}{2}\right)}{\Gamma(6-p)} \frac{(7-p)}{4(6-p)}+\ldots,
\end{aligned}
$$

where $q$ is the momentum of one of the two graviton propagators and the dots stand for subleading terms in $E$. The leading energy result in (B.4) cancels the analogue contribution from the Feynman diagrams of figure 5. The direct evaluation of these diagrams is somewhat involved, in particular for the contribution containing the 3 -graviton vertex. However, as an intermediate check, one can combine the three diagrams, before sewing the common dilaton-dilaton-graviton vertex, and see that, after a Fourier transformation, this reproduces the Dp-brane metric in the Einstein frame at the second order in the $\left(R_{p} / r\right)^{7-p}$ expansion. After including the vertex with the external states, we obtain, at leading order in $E$, a contribution opposite to (B.4). This shows that, in the gauge we chose, all contributions growing with the energy are captured by diagram 4(a). It would be very interesting to check whether a similar pattern holds also at higher orders in $R_{p} / b$ and to see whether the only relevant contributions at high energy are the "half-ladder" diagrams which are a natural generalization of $4(\mathrm{a})$.

\section{Computation of $c_{0}, c_{x}$ and $c_{y}$}

Let us start by showing that $c_{x}$ in (4.17) is vanishing at order $\left(\frac{R_{p}}{b}\right)^{7-p}$. As mentioned in the main text, the first term in the square parenthesis in (4.18) is clearly of higher order, 
since $\ln \sqrt{\alpha} \sim O\left(R_{p} / r\right)^{7-p}$. The second term is a total derivative and its contribution

$$
\left.\partial_{u} \ln \sqrt{\alpha}\right|_{0} ^{\infty}=\left[\frac{C}{\beta} \partial_{r} \ln \sqrt{\alpha}\right]_{r_{*}}^{\infty}=0,
$$

where we first used (4.11) to change the derivative with respect to $u$ into a derivative with respect to $r$ and then used $C\left(r_{*}\right)=0$.

A similar computation holds also for $c_{y}$. The combination $r \sin \bar{\theta}=b+O\left((R / r)^{7-p}\right)$ and so again the first term on the r.h.s. of (4.19) can be neglected at the order $\left(\frac{R_{p}}{b}\right)^{7-p}$. At leading order we have

$$
\left.c_{y} \sim 2 \partial_{u} \ln [\sqrt{\beta} r \sin \bar{\theta}(u)]\right|_{0} ^{\infty}=\left[\frac{C}{\beta} \partial_{r} \ln [\sqrt{\beta} r \sin \theta(r)]\right]_{r_{*}}^{\infty} .
$$

The function $C(r)$ vanishes at the turning point $r_{*}$, so the only possibility for obtaining a non-zero result is to act with the derivative on the $\sin \theta$ term. From (4.2) we see that this yields a factor of $1 / C$ and the lower extremum of (C.2) yields the non-vanishing contribution

$$
\left.2 \frac{b \cos \theta}{r^{2} \beta \sin \theta}\right|_{r=r_{*}}=2 \frac{b \cos \left(\frac{\Theta_{p}+\pi}{2}\right)}{r_{*}^{2} \beta\left(r_{*}\right) \sin \left(\frac{\Theta_{p}+\pi}{2}\right)} \sim-\frac{\Theta_{p}}{b},
$$

where in the first step we used the relation between $\theta\left(r_{*}\right)$ and $\Theta_{p}$ in eq. (4.4) and then we kept only the terms of order $\left(\frac{R_{p}}{b}\right)^{7-p}$. Using (4.8), we obtain the result (4.20).

Let us finally consider the coefficient $c_{0}$. From eq. (4.14) we have

$$
c_{0}=\int_{-\infty}^{+\infty} \frac{\partial_{u}^{2} \sqrt{\beta r^{2}-b^{2} \alpha}}{\sqrt{\beta r^{2}-b^{2} \alpha}} d u
$$

which we can rewrite as

$$
c_{0}=2 \int_{r_{*}}^{\infty} \frac{d r}{\sqrt{\beta r^{2}-b^{2} \alpha}} \partial_{r} \partial_{u} \sqrt{\beta r^{2}-b^{2} \alpha} .
$$

We then compute

$$
\partial_{u} \sqrt{\beta r^{2}-b^{2} \alpha}=\frac{\partial r}{\partial u} \partial_{r} \sqrt{\beta r^{2}-b^{2} \alpha}=\alpha^{-1 / 2}\left(1+\frac{r \beta^{\prime}}{2 \beta}-\frac{b^{2}}{2 r} \frac{\alpha^{\prime}}{\beta}\right),
$$

where we have used eq. (4.11) and a prime denotes differentiation with respect to $r$. Since we are interested only in the lowest order terms that behave as $\left(\frac{R_{p}}{b}\right)^{7-p}$, we can expand $\alpha$ and $\beta$ at large $r$ as follows

$$
\alpha \sim 1+C_{\alpha}\left(\frac{R_{p}}{r}\right)^{7-p}, \quad \beta \sim 1+C_{\beta}\left(\frac{R_{p}}{r}\right)^{7-p} .
$$

We keep the coefficients $C_{\alpha}$ and $C_{\beta}$ arbitrary at this stage, but we will see that the result depends only on the difference $C_{\beta}-C_{\alpha}$, which is equal to one both in the string and in 
the Einstein frame. Substituting eqs. (C.7) in (C.6) and taking the derivative with respect to $r$ we get

$$
\begin{aligned}
\partial_{r}\left[\frac{1}{2}\right. & \left.\left(\frac{R_{p}}{b}\right)^{7-p}\left(-C_{\alpha}+(p-7) C_{\beta}-\frac{b^{2}}{r^{2}}(p-7) C_{\alpha}\right)\right] \\
& =\frac{p-7}{2 r}\left(\frac{R_{p}}{r}\right)^{7-p}\left[-C_{\alpha}+C_{\beta}(p-7)-\frac{b^{2}}{r^{2}} C_{\alpha}(p-9)\right] .
\end{aligned}
$$

We can now insert this expression in eq. (C.5) and make the approximations, valid at the lowest order that we are considering, $r^{*} \sim b$ in the lower extremum of integration and $\alpha$, $\beta \sim 1$ in the square root in the denominator. Using the integral

$$
\int_{b}^{\infty} d r \frac{r^{1-2 \gamma}}{\sqrt{r^{2}-b^{2}}}=\frac{\sqrt{\pi} b^{1-2 \gamma}}{2} \frac{\Gamma\left(\gamma-\frac{1}{2}\right)}{\Gamma(\gamma)},
$$

we obtain

$$
c_{0}=\frac{\sqrt{\pi}}{b}(7-p)\left(\frac{R_{p}}{b}\right)^{7-p} \frac{\Gamma\left(\frac{8-p}{2}\right)}{\Gamma\left(\frac{7-p}{2}\right)}\left(C_{\beta}-C_{\alpha}\right) .
$$

Since $C_{\beta}-C_{\alpha}=1$, the previous result reproduces eq. (4.21).

Open Access. This article is distributed under the terms of the Creative Commons Attribution Noncommercial License which permits any noncommercial use, distribution, and reproduction in any medium, provided the original author(s) and source are credited.

\section{References}

[1] D. Amati, M. Ciafaloni and G. Veneziano, Superstring collisions at Planckian energies, Phys. Lett. B 197 (1987) 81 [SPIRES].

[2] D. Amati, M. Ciafaloni and G. Veneziano, Classical and quantum gravity effects from Planckian energy superstring collisions, Int. J. Mod. Phys. A 3 (1988) 1615 [SPIRES].

[3] D. Amati, M. Ciafaloni and G. Veneziano, Can space-time be probed below the string size?, Phys. Lett. B 216 (1989) 41 [SPIRES].

[4] D. Amati, M. Ciafaloni and G. Veneziano, Higher order gravitational deflection and soft bremsstrahlung in Planckian energy superstring collisions, Nucl. Phys. B 347 (1990) 550 [SPIRES].

[5] D. Amati, M. Ciafaloni and G. Veneziano, Effective action and all order gravitational eikonal at Planckian energies, Nucl. Phys. B 403 (1993) 707 [SPIRES].

[6] B. Sundborg, High-energy asymptotics: the one loop string amplitude and resummation, Nucl. Phys. B 306 (1988) 545 [SPIRES].

[7] G. 't Hooft, Graviton dominance in ultrahigh-energy scattering, Phys. Lett. B 198 (1987) 61 [SPIRES].

[8] I.J. Muzinich and M. Soldate, High-energy unitarity of gravitation and strings, Phys. Rev. D 37 (1988) 359 [SPIRES]. 
[9] D.J. Gross and P.F. Mende, The high-energy behavior of string scattering amplitudes, Phys. Lett. B 197 (1987) 129 [SPIRES].

[10] P.F. Mende and H. Ooguri, Borel summation of string theory for Planck scale scattering, Nucl. Phys. B 339 (1990) 641 [SPIRES].

[11] P.C. Aichelburg and R.U. Sexl, On the gravitational field of a massless particle, Gen. Rel. Grav. 2 (1971) 303 [SPIRES].

[12] M. Fabbrichesi, R. Pettorino, G. Veneziano and G.A. Vilkovisky, Planckian energy scattering and surface terms in the gravitational action, Nucl. Phys. B 419 (1994) 147 [SPIRES].

[13] G. Veneziano, A new approach to semiclassical gravitational scattering, in proceeding of 2nd Journée Cosmolgique, H. J. de Vega and N. Sanchez, eds., pg. 322, talk given at 2nd Journee Cosmologique, Paris, France, 2-4 June (1994), World Scientific Publishing Co., (1995).

[14] D. Amati, M. Ciafaloni and G. Veneziano, Towards an S-matrix description of gravitational collapse, JHEP 02 (2008) 049 [arXiv:0712.1209] [SPIRES].

[15] G. Marchesini and E. Onofri, High energy gravitational scattering: a numerical study, JHEP 06 (2008) 104 [arXiv:0803.0250] [SPIRES].

[16] G. Veneziano and J. Wosiek, Exploring an S-matrix for gravitational collapse, JHEP 09 (2008) 023 [arXiv: 0804.3321] [SPIRES].

[17] G. Veneziano and J. Wosiek, Exploring an S-matrix for gravitational collapse II: a momentum space analysis, JHEP 09 (2008) 024 [arXiv:0805.2973] [SPIRES].

[18] M. Ciafaloni and D. Colferai, S-matrix and quantum tunneling in gravitational collapse, JHEP 11 (2008) 047 [arXiv:0807.2117] [SPIRES].

[19] M. Ciafaloni and D. Colferai, Quantum tunneling and unitarity features of an S-matrix for gravitational collapse, JHEP 12 (2009) 062 [arXiv:0909.4523] [SPIRES].

[20] D.M. Eardley and S.B. Giddings, Classical black hole production in high-energy collisions, Phys. Rev. D 66 (2002) 044011 [gr-qc/0201034] [SPIRES].

[21] E. Kohlprath and G. Veneziano, Black holes from high-energy beam-beam collisions, JHEP 06 (2002) 057 [gr-qc/0203093] [SPIRES].

[22] H. Yoshino and Y. Nambu, Black hole formation in the grazing collision of high-energy particles, Phys. Rev. D 67 (2003) 024009 [gr-qc/0209003] [SPIRES].

[23] S.B. Giddings and V.S. Rychkov, Black holes from colliding wavepackets, Phys. Rev. D 70 (2004) 104026 [hep-th/0409131] [SPIRES].

[24] G. Veneziano, String-theoretic unitary S-matrix at the threshold of black-hole production, JHEP 11 (2004) 001 [hep-th/0410166] [SPIRES].

[25] S.B. Giddings, Locality in quantum gravity and string theory, Phys. Rev. D 74 (2006) 106006 [hep-th/0604072] [SPIRES].

[26] M.J. Duff, Quantum tree graphs and the Schwarzschild solution, Phys. Rev. D 7 (1973) 2317 [SPIRES].

[27] M.J. Duff, Quantum corrections to the Schwarzschild solution, Phys. Rev. D 9 (1974) 1837 [SPIRES].

[28] J. Polchinski, Dirichlet-branes and Ramond-Ramond charges, Phys. Rev. Lett. 75 (1995) 4724 [hep-th/9510017] [SPIRES]. 
[29] G.T. Horowitz and A. Strominger, Black strings and P-branes, Nucl. Phys. B 360 (1991) 197 [SPIRES].

[30] M.R. Garousi and R.C. Myers, Superstring scattering from D-branes, Nucl. Phys. B 475 (1996) 193 [hep-th/9603194] [SPIRES].

[31] P. Di Vecchia et al., Classical p-branes from boundary state, Nucl. Phys. B 507 (1997) 259 [hep-th/9707068] [SPIRES].

[32] M. Bertolini et al., Is a classical description of stable non-BPS D-branes possible?, Nucl. Phys. B 590 (2000) 471 [hep-th/0007097] [SPIRES].

[33] M. Fabbrichesi and R. Iengo, Scattering of a massless particle by the 'sun' in superstring theory, Phys. Lett. B 264 (1991) 319 [SPIRES].

[34] J. Polchinski, String theory. Vol. 2: Superstring theory and beyond, Cambridge University Press, Cambridge U.K. (1998) pg. 531.

[35] I.R. Klebanov and L. Thorlacius, The size of p-branes, Phys. Lett. B 371 (1996) 51 [hep-th/9510200] [SPIRES].

[36] A. Hashimoto and I.R. Klebanov, Scattering of strings from D-branes, Nucl. Phys. Proc. Suppl. 55B (1997) 118 [hep-th/9611214] [SPIRES].

[37] M. Ademollo et al., Unified dual model for interacting open and closed strings, Nucl. Phys. B 77 (1974) 189 [SPIRES].

[38] H.D.I. Abarbanel and C. Itzykson, Relativistic eikonal expansion, Phys. Rev. Lett. 23 (1969) 53 [SPIRES].

[39] M. Levy and J. Sucher, Eikonal approximation in quantum field theory, Phys. Rev. 186 (1969) 1656 [SPIRES].

[40] P. Di Vecchia and A. Liccardo, D-branes in string theory. 1., NATO Adv. Study Inst. Ser. C. Math. Phys. Sci. 556 (2000) 1, [hep-th/9912161] [SPIRES].

[41] A. Pasquinucci, On the scattering of gravitons on two parallel D-branes, Mod. Phys. Lett. A 12 (1997) 1537 [hep-th/9703066] [SPIRES].

[42] S. Lee and S.-J. Rey, Absorption and recoil of fundamental string by D-string, Nucl. Phys. B 508 (1997) 107 [hep-th/9706115] [SPIRES].

[43] V. Alessandrini, D. Amati and B. Morel, The asymptotic behaviour of the dual Pomeron amplitude, Nuovo Cim. A 7 (1972) 797 [SPIRES].

[44] E. Gava, R. Iengo and C.-J. Zhu, Quantum gravity corrections from superstring theory, Nucl. Phys. B 323 (1989) 585 [SPIRES].

[45] R. Iengo and K. Lechner, Schwarzschild like corrections to gravity from superstrings at one loop, Nucl. Phys. B 335 (1990) 221 [SPIRES].

[46] S.B. Giddings and M. Srednicki, High-energy gravitational scattering and black hole resonances, Phys. Rev. D 77 (2008) 085025 [arXiv:0711.5012] [SPIRES].

[47] S.B. Giddings and R.A. Porto, The gravitational S-matrix, Phys. Rev. D 81 (2010) 025002 [arXiv: 0908.0004] [SPIRES].

[48] G. Veneziano, Strings and gravitation, in proceedings of 5th Marcel Grossmann Meeting "Recent developments in theoretical and experimental general relativity, gravitation and relativistic field theories", D.G. Blair and M.J. Buckingham eds., Perth (1988) pg. 173. 
[49] H.J. de Vega and N.G. Sanchez, Quantum string scattering in the Aichelburg-Sexl geometry, Nucl. Phys. B 317 (1989) 706 [SPIRES].

[50] G.T. Horowitz and A.R. Steif, Strings in strong gravitational fields, Phys. Rev. D 42 (1990) 1950 [SPIRES].

[51] S.B. Giddings, D.J. Gross and A. Maharana, Gravitational effects in ultrahigh-energy string scattering, Phys. Rev. D 77 (2008) 046001 [arXiv:0705.1816] [SPIRES].

[52] M. Blau, J.M. Figueroa-O'Farrill and G. Papadopoulos, Penrose limits, supergravity and brane dynamics, Class. Quant. Grav. 19 (2002) 4753 [hep-th/0202111] [SPIRES].

[53] J.L.F. Barbon, D-brane form factors at high energy, Phys. Lett. B 382 (1996) 60 [hep-th/9601098] [SPIRES].

[54] C. Bachas and B. Pioline, High-energy scattering on distant branes, JHEP 12 (1999) 004 [hep-th/9909171] [SPIRES].

[55] W. Black and C. Monni, work in progress.

[56] S.D. Mathur, The fuzzball proposal for black holes: An elementary review, Fortsch. Phys. $\mathbf{5 3}$ (2005) 793 [hep-th/0502050] [SPIRES].

[57] S. Giusto, J.F. Morales and R. Russo, D1D5 microstate geometries from string amplitudes, JHEP 03 (2010) 130 [arXiv:0912.2270] [SPIRES].

[58] W. Black, R. Russo and D. Turton, The supergravity fields for a D-brane with a travelling wave from string amplitudes, Phys. Lett. B 694 (2010) 246 [arXiv:1007.2856] [SPIRES].

[59] A. Strominger and C. Vafa, Microscopic origin of the Bekenstein-Hawking entropy, Phys. Lett. B 379 (1996) 99 [hep-th/9601029] [SPIRES].

[60] T. Huber and D. Maître, HypExp 2, expanding hypergeometric functions about half-integer parameters, Comput. Phys. Commun. 178 (2008) 755 [arXiv: 0708. 2443] [SPIRES]. 